\title{
Structural basis of broad ebolavirus neutralization by a human survivor antibody
}

\author{
Brandyn R. West 1,12, Anna Z. Wec 2,10,12, CrystalL. Moyer ${ }^{1,11}$, Marnie L. Fusco', Philipp A. Ilinykh ${ }^{3,4}$, \\ Kai Huang ${ }^{3,4}$, Ariel S. Wirchnianski², Rebekah M. James ${ }^{5}$, Andrew S. Herbert5, Sean Hui ${ }^{1}$, \\ Eileen Goodwin ${ }^{6}$, Katie A. Howell7, Shweta Kailasan7, M. Javad Aman7, Laura M. Walker ${ }^{6}$, John M. Dye ${ }^{5}$, \\ Alexander Bukreyev ${ }^{3,4,8}$, Kartik Chandran ${ }^{2 \star}$ and Erica Ollmann Saphire ${ }^{10}{ }^{1,9 \star}$
}

The structural features that govern broad-spectrum activity of broadly neutralizing anti-ebolavirus antibodies (Abs) outside of the internal fusion loop epitope are currently unknown. Here we describe the structure of a broadly neutralizing human monoclonal Ab (mAb), ADI-15946, which was identified in a human survivor of the 2013-2016 outbreak. The crystal structure of ADI-15946 in complex with cleaved Ebola virus glycoprotein (EBOV $\mathrm{GP}_{\mathrm{CL}}$ ) reveals that binding of the $\mathrm{mAb}$ structurally mimics the conserved interaction between the EBOV GP core and its glycan cap $\beta 17-\beta 18$ loop to inhibit infection. Both endosomal proteolysis of EBOV GP and binding of mAb FVM09 displace this loop, thereby increasing exposure of ADI-15946's conserved epitope and enhancing neutralization. Our work also mapped the paratope of ADI-15946, thereby explaining reduced activity against Sudan virus, which enabled rational, structure-guided engineering to enhance binding and neutralization of Sudan virus while retaining the parental activity against EBOV and Bundibugyo virus.

E $\mathrm{BOV}$ and related members of the family Filoviridae cause outbreaks of highly lethal disease in humans. Ab therapeutics such as ZMapp ${ }^{1}, \mathrm{mAb}_{114^{2}}$ and a three-mAb cocktail from Regeneron Pharmaceuticals (REGN-EB3) ${ }^{3}$ have recently been proposed for emergency use against Ebola virus disease ${ }^{4}$. However, the activity of these therapies is limited to EBOV and does not extend protection to the related virulent ebolaviruses Bundibugyo virus (BDBV) and Sudan virus (SUDV). Both BDBV and SUDV have caused sizeable outbreaks in the past and their potential for re-emergence remains unknown. No therapeutics are currently available for the treatment of BDBV and SUDV, which lends urgency to discovery and characterization of broadly active mAbs. The key obstacle for generation of such mAbs stems from limited amino acid sequence conservation among the glycoproteins of ebolaviruses, with only $50 \%$ amino acid identity shared between EBOV and SUDV, the two most prevalent ebolaviruses. Detailed characterization of known broadly neutralizing antibodies (bNAbs) and their modes of action will be critical to the design of next-generation broadly protective Ab cocktails and vaccines that elicit broadly protective responses.

All $\mathrm{Ab}$ therapeutics currently under development for Ebola virus disease target the ebolavirus surface glycoprotein, GP, which mediates viral entry into host cells by catalyzing viral membrane fusion in host cell endosomes ${ }^{1,2,5}$. During biogenesis, GP is posttranslationally processed to yield GP1 and GP2 subunits (Fig. 1a), held together by a single disulfide bond, which associate into a trimer of GP1,2 heterodimers ${ }^{6,7}$. GP1 mediates host cell attachment and receptor recognition, whereas GP2 mediates fusion of the viral and host membranes ${ }^{8-12}$. The GP2 amino acid sequence, which includes the internal fusion loop (IFL), is highly conserved among ebolaviruses ${ }^{6}$ while the glycan cap and mucin-like domains of GP1 show higher sequence diversity. During infection, EBOV GP undergoes host-programmed disassembly mediated by endosomal cysteine proteases (cathepsins B and L), which shed the steric bulk of the extensively glycosylated glycan cap and mucin-like domains and generate the cleaved GP intermediate $\left(\mathrm{GP}_{\mathrm{CL}}\right)^{13,14}$. This in turn unmasks the receptor-binding site in GP1 and reveals other previously inaccessible regions of the GP core ${ }^{12,15,16}$. Engagement of the intracellular entry receptor Niemann-Pick C1 (NPC1) by the viral receptor-binding site is proposed to induce conformational rearrangements in $\mathrm{GP}_{\mathrm{CL}}$ that culminate in viral membrane fusion ${ }^{12,16-19}$. Antibodies targeting the functionally critical and conserved viral fusion machinery offer an efficacious mode of viral entry inhibition and have been shown to be strongly protective $\mathrm{e}^{20-24}$.

The only pan-ebolavirus neutralizing Abs reported thus far target overlapping epitopes in the viral IFL ${ }^{21,23-26}$. In contrast, lowresolution negative stain reconstructions of the recently isolated human survivor mAb, ADI-15946, suggest that it may recognize a distinct footprint in the base region of GP, crosslinking the GP1 and GP2 subunits ${ }^{20}$. ADI-15946 potently neutralizes EBOV and BDBV but lacks neutralizing and protective activity against $\mathrm{SUDV}^{20}$. Accordingly, we sought to define the structural determinants of ADI-15946 activity in the context of its cognate antigen, EBOV GP, to characterize features of both its epitope and paratope that confer broad reactivity while investigating its limited activity against SUDV.

\footnotetext{
'Department of Immunology and Microbiology, The Scripps Research Institute, La Jolla, CA, USA. ²Department of Microbiology and Immunology, Albert Einstein College of Medicine, Bronx, NY, USA. ${ }^{3}$ Department of Pathology, University of Texas Medical Branch, Galveston, TX, USA. ${ }^{4}$ Galveston National Laboratory, University of Texas Medical Branch, Galveston, TX, USA. 'Division of Virology, United States Army Medical Research Institute of Infectious Diseases, Fort Detrick, MD, USA. ${ }^{6}$ Adimab LLC, Lebanon, NH, USA. ${ }^{7}$ Integrated Biotherapeutics, Rockville, MD, USA. ${ }^{8}$ Department of Immunology and Microbiology, University of Texas Medical Branch, Galveston, TX, USA. ' $5 k a g g s$ Institute for Chemical Biology, The Scripps Research Institute, La Jolla, CA, USA. ${ }^{10}$ Present address: Adimab LLC, Lebanon, NH, USA. "1Present address: Mapp Biopharmaceutical, San Diego, CA, USA. ${ }^{12}$ These authors contributed equally: Brandyn R. West, Anna Z.Wec. *e-mail: kartik.chandran@einstein.yu.edu; erica@scripps.edu
} 


\section{Results}

Crystal structure of EBOV GP $\mathrm{CL}-\mathrm{ADI}-15946$ complex. We determined the crystal structure of ADI-15946's fragment antigen binding (Fab) in complex with EBOV GP $\mathrm{CL}_{\mathrm{L}}$ to $4.1 \AA$ resolution (Fig. 1a,b and Table 1$)^{20}$. The structure was solved by molecular replacement using the previously published EBOV $\mathrm{GP}_{\mathrm{CL}}$ structure as a search model (PDB 5HJ3) and was refined to an $R_{\text {work }} / R_{\text {free }}$ of $26.2 / 28.1 \%$ (Table 1$)^{16}$. The ADI-15946 Fab targets the base of a single GP1/GP2 protomer in the GP trimer at an approximately $45^{\circ}$ angle relative to the surface of GP, with its constant domains directed downward toward the viral membrane (Fig. 1b).

The heavy and light chains each contribute roughly half of the overall ADI-15946 binding interface: $~ 55 \%$ from the heavy chain complementarity-determining region 3 (CDR H3), and the remaining $45 \%$ from the combined contributions of the light chain framework region 3 (FRL3) and CDRs 1, 2 and 3 (L1, L2 and L3, respectively) (Fig. 1b,c). Notably, CDRs 1 and 2 of the heavy chain ( $\mathrm{H} 1$ and $\mathrm{H} 2$, respectively) do not participate in the Fab-GP $\mathrm{CL}$ interface (Fig. 1b,c). Instead, heavy chain recognition of $\mathrm{GP}_{\mathrm{CL}}$ is mediated exclusively by the CDR $\mathrm{H} 3$ that is 22 amino acids long, which buries roughly $465 \AA^{2}$ of surface area on binding (Fig. 1b). The footprint of ADI-15946 partially overlaps those of the EBOVmonospecific binders KZ52, c2G4 and c4G7 (Supplementary Note 1$)^{6,27}$. However, ADI-15946 probably gains cross-reactivity from an upward shift of its footprint that allows it to target a highly conserved pocket above the upper boundary of the KZ52 epitope as suggested previously (Fig. 1d) ${ }^{20}$.

Modeling ADI-15946 interactions with GP2 and the glycan cap. ADI-15946 heavy chain contacts include the strictly conserved lysine at position 510 in the GP2 $\mathrm{N}$ terminus-where a substitution to glutamic acid, $\mathrm{K} 510 \mathrm{E}$, was previously shown to give rise to escape from BDBV viral neutralization ${ }^{20}$. We have confirmed that the same amino acid substitution in EBOV GP also leads to the loss of binding and neutralization phenotypes (data not shown). The electrostatic surface of ADI-15946 is complementary to that of GP, and our sidechain modeling suggests that the K510E substitution introduces a charged/steric clash with residues $\mathrm{D} 100^{\mathrm{C}}$ and/or $\mathrm{L} 100^{\mathrm{F}}$ of CDR $\mathrm{H} 3$ thereby explaining the loss of antiviral activity (Fig. 2a-c). We tested ADI-15946 variants containing alanine substitutions at either the $\mathrm{D} 100^{\mathrm{C}}$ or the $\mathrm{L} 100^{\mathrm{F}}$ positions for binding and neutralization and observed loss of both activities against recombinant vesicular stomatitis virus (rVSV) particles bearing BDBV GP K510E (Fig. 2d). Additionally, the ADI-15946 D100 ${ }^{\mathrm{C}} \mathrm{A}$ variant lost activity to wild-type rVSV-BDBV GP probably due to the loss of stabilizing interactions between the $\mathrm{D} 100^{\mathrm{C}}$ sidechain and the backbone of CDR H3 (Fig. 2d). These results indicated that interaction with the conserved K510 residue is a key contributor to ADI-15946's breadth and that overcoming the K510E escape mutation would be difficult without extensive mutagenesis of the antibody paratope.

Fig. 1 | Structure of ADI-15946 in complex with ebolavirus $\mathrm{GP}_{\mathrm{CL}}$. a, Domain architecture of EBOV GP full length and the construct crystallized here, which is the ectodomain of an enzymatically cleaved $\mathrm{GP}\left(\mathrm{GP}_{\mathrm{CL}}\right)$ resembling the form of $\mathrm{GP}$ generated in endosomes during viral entry lacking the glycan cap. HR1 and HR2, heptad repeat 1 and heptad repeat 2, respectively. Glycosylation sites are represented above the domains. b, Crystal structure of the trimeric EBOV GP $\mathrm{CL}_{\mathrm{C}}-\mathrm{ADI}-15946$ complex. The inset table shows the contribution of each complementaritydetermining region (CDR) to the buried surface area (BSA) on the surface of $\mathrm{GP}_{\mathrm{CL}}$. $\mathbf{c}$, The interaction bridges the fusion loop and other portions of GP2 primarily via light chain and $\mathrm{CDR} H 3$ contacts. $\mathrm{CDRs} H 1$ and $\mathrm{H} 2$ are not involved. d, The footprint of ADI-15946 (orange) is shifted up and to the left compared to that of the base-binding mAb, KZ52 (pink).
Wec et al. ${ }^{20}$ have shown that ADI-15946 inhibits GP proteolysis in vitro, a key step in unmasking of the viral receptor-binding site that is indispensable for infection. The left boundary of ADI15946's footprint, formed by light chain FRL3, is in immediate proximity to the $\beta 13-\beta 14$ loop that passes over the IFL and is cleaved by endosomal cathepsins during viral entry (Supplementary Fig. 1) ${ }^{13,14}$.
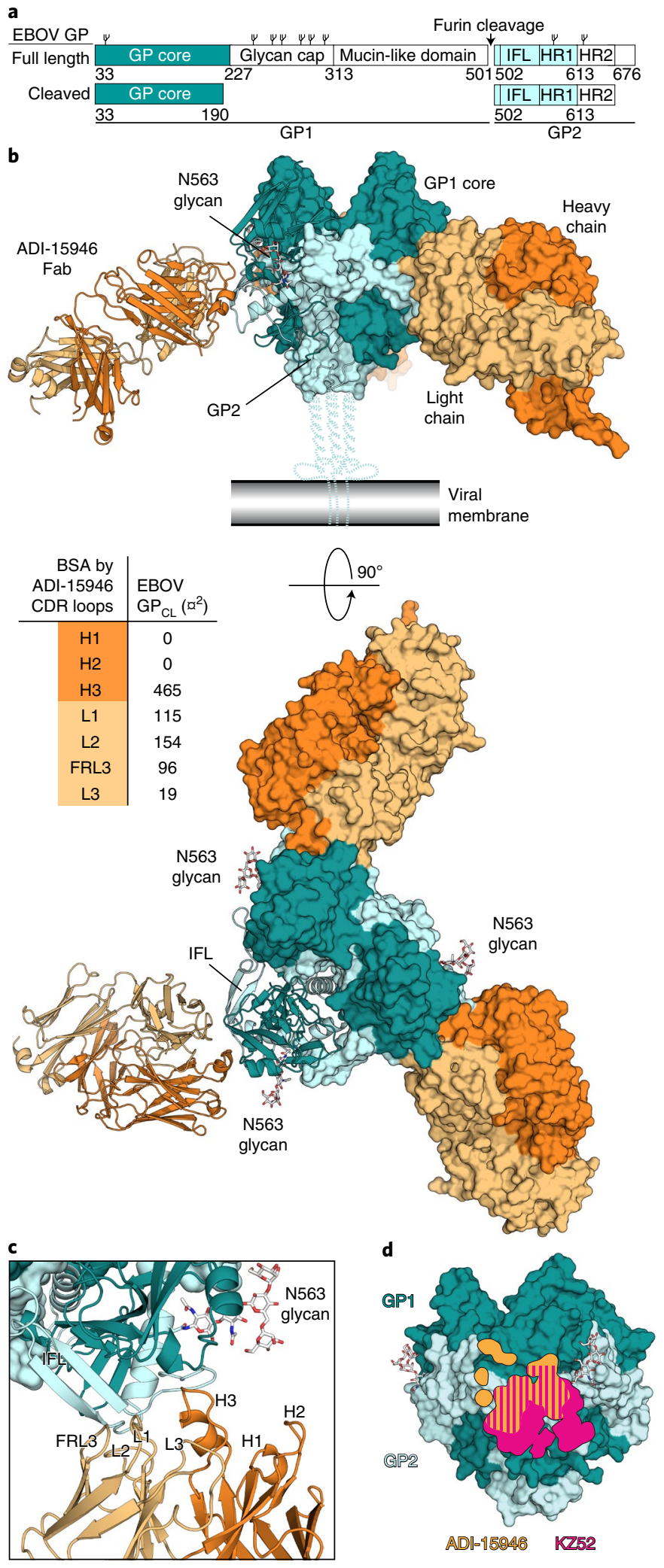
Table 1 | Data collection and refinement statistics

\section{EBOV GP ${ }_{\mathrm{CL}}-\mathrm{ADI}-15946 \mathrm{Fab}$ (PDB} 6MAM)

\begin{tabular}{ll}
\hline $\begin{array}{l}\text { Data collection } \\
\text { Space group }\end{array}$ & \\
$\begin{array}{l}\text { Cell dimensions } \\
\quad a, b, c(\AA)\end{array}$ & $182.27,182.27,262.01$ \\
$\quad \alpha, \beta, \gamma\left(^{\circ}\right)$ & $90,90,90$ \\
Resolution $(\AA)$ & $48.54-4.10(4.25-4.10)^{\mathrm{b}}$ \\
$R_{\text {merge }}$ & $0.20(1.83)$ \\
$R_{\text {pim }}$ & $0.05(0.46)$ \\
$I / \sigma(I)$ & $14.6(1.8)$ \\
$C C_{1 / 2}$ & $1.00(0.70)$ \\
Completeness $(\%)$ & $99.6(100.0)$ \\
Redundancy & $13.1(13.4)$ \\
\end{tabular}

\section{Refinement}

Resolution $(\AA)$

$48.54-4.10(4.25-4.10)$

No. reflections

$35,153(3,458)$

$R_{\text {work }} / R_{\text {free }}$

$0.262 / 0.281$

No. atoms

Protein

Ligands (glycans)

$B$ factors

Protein 114

Ligands (glycans) $\quad 107$

R.m.s. deviations

Bond lengths $(\AA)$

0.01

Bond angles $\left({ }^{\circ}\right)$

0.61

aDiffraction data were collected from a single crystal. 'balues in parentheses are for highestresolution shell.

Overlay of our structure with uncleaved GP (PDB 5JQ3) shows that ADI-15946 binding probably impedes protease access to this loop, thus explaining ADI-15946 inhibition of GP proteolysis (Supplementary Fig. 1) ${ }^{20}$.

Competition with, and mimicry of, the $\beta 17-\beta 18$ loop. ADI15946 binds into a hydrophobic pocket formed by residues $71-75$ of GP1, which fold into a $3_{10}$ helix, and is hereafter termed the ' 3 10 pocket' (Fig. 3a,b). The $3_{10}$ helix rearranges in $\mathrm{GP}_{\mathrm{CL}}$ when bound to NPC1, and stabilization of this region by ADI-15946 CDR H3 may be part of the mAb's neutralization mechanism (Fig. 3a) ${ }^{12,16,20}$. In uncleaved GP, the $3_{10}$ pocket is occupied by the $\beta 17-\beta 18$ loop (GP1 residues 287-291) that extends down from the glycan cap (Fig. 3a,b) ${ }^{12,28}$. Interactions of ADI-15946 CDR H3 residues D100', $\mathrm{W} 100^{\mathrm{D}}$ and $\mathrm{L} 100^{\mathrm{E}}$ with the $3_{10}$ pocket mimic the contacts made by $\beta 17-\beta 18$ loop residues E292, W291, and F290, respectively (Fig. 3b). Correspondingly, we postulated that the viral $\beta 17-\beta 18$ loop peptide competes for binding with CDR H3 of ADI-15946 in full-length GP $\left(\mathrm{GP}_{\mathrm{FL}}\right)$ (Supplementary Fig. 2 ). To test the effect of $\beta 17-\beta 18$ loop removal on ADI-15946 binding and neutralization, we measured the apparent binding constant $\left(K_{\mathrm{d}}^{\text {app }}\right)$ for ADI-15946 interaction with EBOV $\mathrm{GP}_{\mathrm{FL}}$ and $\mathrm{GP}_{\mathrm{CL}}$ via biolayer interferometry, as well as its neutralization of rVSV-EBOV GP $\mathrm{FL}_{\mathrm{F}}$ and rVSV-EBOV $\mathrm{GP}_{\mathrm{CL}}$. Consistent with the previous report, we observed a 10,000-fold improvement in binding and a 100 -fold improvement in neutralization potency against EBOV $\mathrm{GP}_{\mathrm{CL}}$ compared to full-length $\mathrm{GP}$ (Fig. $3 \mathrm{e}$ and Supplementary Tables 1 and 2$)^{20}$. Comparison of association $\left(k_{\mathrm{on}}\right)$ and dissociation $\left(k_{\text {off }}\right)$ rates of $\mathrm{ADI}-15946$ to $\mathrm{EBOV} \mathrm{GP}_{\mathrm{FL}}$ and $\mathrm{GP}_{\mathrm{CL}}$ showed that the improved binding to $\mathrm{GP}_{\mathrm{CL}}$ is primarily driven by an over 1,000-fold slower $k_{\text {off }}$ and a modest improvement of the association rate (10-fold increased $k_{\text {on }}$ ) (Fig. 3f, Supplementary Fig. 3 and Supplementary Table 2). Given the sizeable contribution of CDR H3 to the binding interface, the slower dissociation rate against $\mathrm{GP}_{\mathrm{CL}}$ probably results from its unobstructed access to the $3_{10}$ pocket.

To specifically probe the importance of the $\beta 17-\beta 18$ loop's hydrophobic packing into the $3_{10}$ pocket to the shielding of the ADI15946 epitope, we first tested ADI-15946 binding and neutralization against rVSVs bearing an EBOV GP variant with an arginine substitution at position $291\left(\mathrm{rVSV}-E B O V \mathrm{GP}_{\mathrm{W} 291 \mathrm{R}}\right)$. Modeling of the arginine sidechain suggested that the W291R substitution would displace the $\beta 17-\beta 18$ loop from the conserved pocket by introducing charged and steric clashes with the GP2 residue asparagine 512. Consistent with this hypothesis, we observed a 10-fold enhancement in ADI-15946 binding and neutralization of rVSV-EBOV GP ${ }_{\mathrm{W} 291 \mathrm{R}}$ compared to rVSV bearing wild-type EBOV GP (GP ${ }_{\mathrm{WT}}$ ) (Fig. 3d,e and Supplementary Table 1). We then tested ADI-15946 neutralization against $r V S V$ s bearing an EBOV GP $\Delta \beta 17-\beta 18$ variant in which the $\beta 17-\beta 18$ loop has been deleted (residues 187-198). Removal of the $\beta 17-\beta 18$ loop had no apparent adverse effects on viral infectivity or replication in vitro (data not shown). We observed a 10 -fold increase in neutralization efficiency of rVSV-EBOV GP $\Delta \beta 17$ $\beta 18$ compared to $\mathrm{GP}_{\mathrm{WT}}$ (Fig. $3 \mathrm{c}$ ). The intermediate neutralization potency of rVSV-EBOV $\mathrm{GP}_{\mathrm{W} 291 \mathrm{R}}$ compared to $\mathrm{GP}_{\mathrm{CL}}$ and $\mathrm{GP}_{\mathrm{WT}}$ suggests that displacement of the $\beta 17-\beta 18$ loop from the $3_{10}$ pocket is the limiting factor for binding of ADI-15946 to EBOV GP (Fig. 3e).

Enhancement of binding and neutralization with mAb FVM09. Previous work suggests that a non-neutralizing mAb, FVM09, recognizes and 'peels away' the $\beta 17-\beta 18$ loop from the base, thereby enhancing neutralization by the base-binding mAb c2 $\mathrm{G}^{29,30}$. Given the more profound role of the $\beta 17-\beta 18$ loop in restricting access of ADI-15946 to its base epitope (see above), we evaluated the hypothesis that FVM09 could also work together with ADI-15946 to neutralize virus with enhanced potency (Supplementary Fig. 2). We first tested competition between ADI-15946 and FVM09 using biolayer interferometry-based competitive binding assays and found that both mAbs could bind to EBOV GP simultaneously (Supplementary Fig. 4). We next tested the effect of FVM09 on ADI-15946 neutralization of rVSV-EBOV GP or authentic EBOV in the presence of three fixed concentrations of FVM09. FVM09 potentiated ADI-15946 neutralization in a concentration-dependent manner by $>10$-fold, but had no effect on neutralization by KZ52 (Fig. 4a). Conversely, titration of FVM09 into a constant, subneutralizing concentration of ADI-15946 also showed dose-dependent enhancement of ADI15946 neutralizing activity (Fig. 4b). In contrast, KZ52 neutralization was reduced in the same assay, perhaps because KZ52 binds along the surface of the $\beta 17-\beta 18$ loop whereas ADI- 15946 binds beneath the $\beta 17-\beta 18$ loop (Fig. $4 \mathrm{~b}$ and Supplementary Fig. 5). We observed similar neutralization enhancement trends with authentic EBOV (Fig. 4c,d), and to a lesser extent, with rVSV-SUDV GP (Fig. 4e,f). The functional importance of the strict conservation of the amino acid sequence in the $3_{10}$ pocket and the $\beta 17-\beta 18$ loop among ebolavirus GPs remains unknown, but it may relate to the transduction of conformational changes in GP on NPC1 binding (Fig. $3 \mathrm{a}$ and Supplementary Note 2$)^{12}$. Our results demonstrate that rare neutralizing antibodies such as ADI-15946 can access and exploit this cryptic site of broad ebolavirus vulnerability to achieve neutralization, presumably by interfering with the region's functional role in viral entry. The enhancement of ADI-15946 binding and neutralization in the presence of $\beta 17-\beta 18$ loop binders such as FVM09 may reflect a mechanism by which specific combinations of glycan cap- and base-binding mAbs can synergize to interdict viral infection during a natural polyclonal immune response (Fig. 4g). 
a

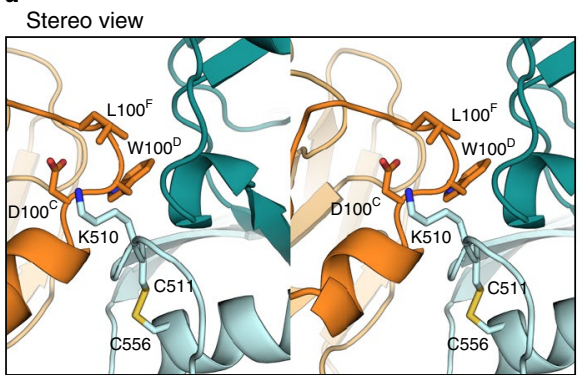

b

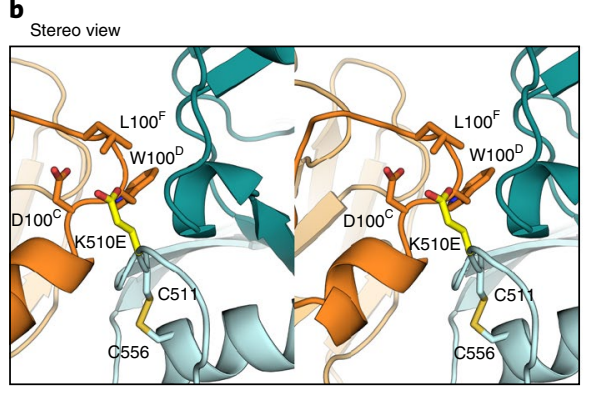

c

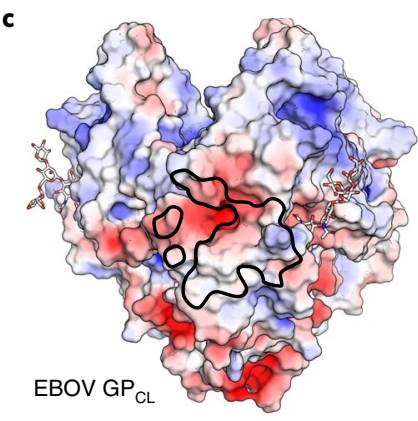

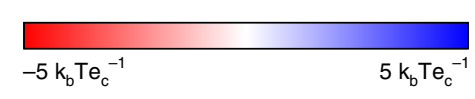

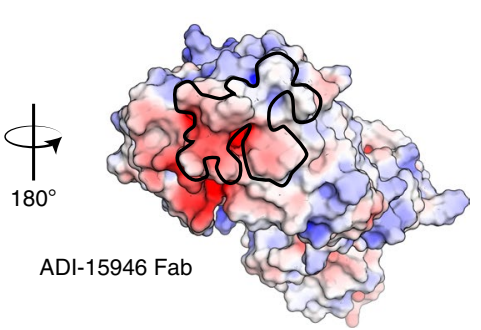

d
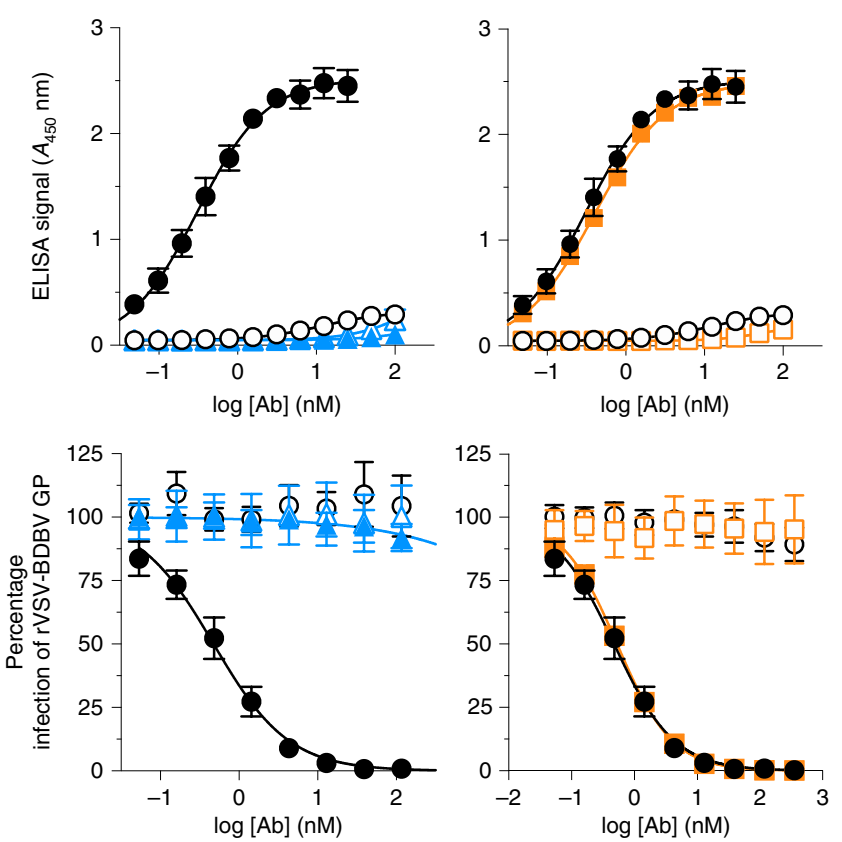

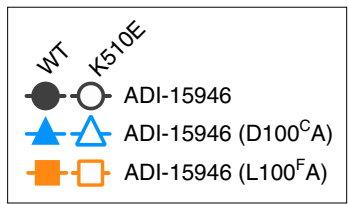

Fig. 2 | The K510E escape mutation probably clashes with ADI-15946 CDR H3. a, Stereoview of the EBOV GP $\mathrm{CL}^{-A D I-15946 ~ c o m p l e x ~(l e f t ; ~ A D I-15946 ~}$ in orange, and $\mathrm{GP}_{\mathrm{CL}}$ in dark and light teal for GP1 and GP2, respectively) and electrostatic surface potential (right; color scale shown in c) showing that residue $\mathrm{K} 510$ of GP2 binds into a negatively charged pocket created by ADI-15946 CDR H3. b, Similar views to a, with modeling of an escape mutant of ADI-15946, GP K510E, suggesting that K510E clashes with CDR H3 and introduces conflicting negative charge into the CDR H3 pocket. c, Open-book representation of EBOV GP $P_{C L}$ and $A D I-15946$ showing electrostatic surface potential colored according to included scale. $\mathrm{GP}_{\mathrm{CL}}$ is shown on the left with the epitope outlined in black. ADI-15946 is shown on the right with the paratope outlined in black. $\mathrm{k}_{\mathrm{b}}$, Boltzmann's constant $\left(1.3806504 \times 10^{-23} \mathrm{~J} \mathrm{~K} \mathrm{~K}^{-1}\right)$.

$\mathrm{T}$, temperature $\left(310{ }^{\circ} \mathrm{K}\right)$. $\mathrm{e}_{c^{\prime}}$ charge of an electron $\left(1.60217646 \times 10^{-19} \mathrm{C}\right)$. d, Binding and neutralization assays showing the capacity of ADI-15946 variants containing either the D100 A or the L100FA mutation to bind to rVSV-BDBV GP (wild type, WT or K510E) in an ELISA (top, mean \pm s.d., $n=4$ biologically independent samples) and neutralize infection by these viruses (bottom, mean \pm s.d., $n=6$ biologically independent samples). Electrostatic surface potentials in a and $\mathbf{c}$ were generated using the APBS plugin with Pymol. $A_{450} \mathrm{~nm}$, absorbance at $450 \mathrm{~nm}$.

Molecular determinants for somatic maturation of ADI-15946. To delineate the molecular basis of ADI-15946's broad activity, we assigned its light and heavy chain variable domain sequences to their most probable inferred germline progenitors (IGL) (Fig. 5a and Supplementary Note 3). We found that reversion of three residues introduced by somatic hypermutation (SHM) in the variable heavy chain domain to germline (light chain, LC; heavy chain, HC) $\left(\mathrm{LC}^{\mathrm{WT}}: \mathrm{HC}^{\mathrm{IGL}}\right.$; CDR H3 is retained fully mature) had no appreciable impact on binding of full-length or cleaved EBOV, BDBV or SUDV GP or neutralization of rVSVs bearing EBOV, BDBV or SUDV GP (Fig. 5b,c and Supplementary Fig. 6). These findings agree with our structural observations, since only CDR H3 participates in binding to $\mathrm{EBOV} \mathrm{GP}_{\mathrm{CL}}$ (Fig. 1b).

Reversion of 10 SHM-introduced residues in the variable heavy chain domain outside of CDR L3 (LC ${ }^{\mathrm{IGL}}: \mathrm{HC}^{\mathrm{WT}}$; residues 3, 4, 27, $28,31,53,67,72,87,104)$ had a profound impact on neutralization of rVSVs bearing EBOV, BDBV or SUDV GP (Fig. 5b,c and Supplementary Fig. 6). Both the LC ${ }^{\mathrm{IGL}}: \mathrm{HC}^{\mathrm{WT}}$ and the combined $\mathrm{LC}^{\mathrm{IGL}}: \mathrm{HC}{ }^{\mathrm{IGL}} \mathrm{Ab}$ variants were non-neutralizing against $\mathrm{rVSV}-\mathrm{EBOV}$
$\mathrm{GP}_{\mathrm{FL}}$ despite retaining a fully mature $\mathrm{CDR} \mathrm{H} 3$ and $\mathrm{CDR} \mathrm{L} 3$, suggesting that LC contacts outside of CDR L3 make key contributions to GP recognition (Fig. 5c). The $\mathrm{LC}^{\mathrm{IGL}}: \mathrm{HC}^{\mathrm{WT}}$ and the $\mathrm{LC}^{\mathrm{IGL}}: \mathrm{HC}^{\mathrm{IGL}}$ germline-approximating $\mathrm{Ab}$ variants retained some neutralizing activity against $\mathrm{EBOV} \mathrm{GP}_{\mathrm{CL}}$ and $\mathrm{BDBV} \mathrm{GP}_{\mathrm{CL}}$, probably due to improved access of CDR $\mathrm{H} 3$ to the $3_{10}$ pocket (Fig. $5 \mathrm{c}$ and Supplementary Table 1). However, LC ${ }^{\mathrm{IGL}}: \mathrm{HC}^{\mathrm{IGL}}$ did not neutralize

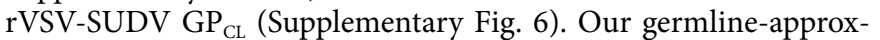
imating variants $\mathrm{LC}^{\mathrm{IGL}}: \mathrm{HC}^{\mathrm{WT}}$ and the $\mathrm{LC}^{\mathrm{IGL}}: \mathrm{HC}^{\mathrm{IGL}}$ neutralized the rVSV-EBOV $\mathrm{GP}_{\mathrm{W} 291 \mathrm{R}}$ variant more potently than rVSV-EBOV $\mathrm{GP}_{\mathrm{CL}}$, which suggests that additional LC contacts that are absent in $\mathrm{GP}_{\mathrm{CL}}$, probably between the $\mathrm{Ab}$ and the glycan cap, contribute to ADI-15946's activity against GP ${ }_{\mathrm{FL}}$ (Supplementary Figs. 1 and 6 and Supplementary Table 1).

Enhancement of ADI-15946 activity against SUDV. Our analysis of the conservation in ADI-15946's epitope shows that limited SUDV reactivity and lack of activity against Reston virus (RESTV) probably arise from the amino acid sequence divergence on the edges of 
a
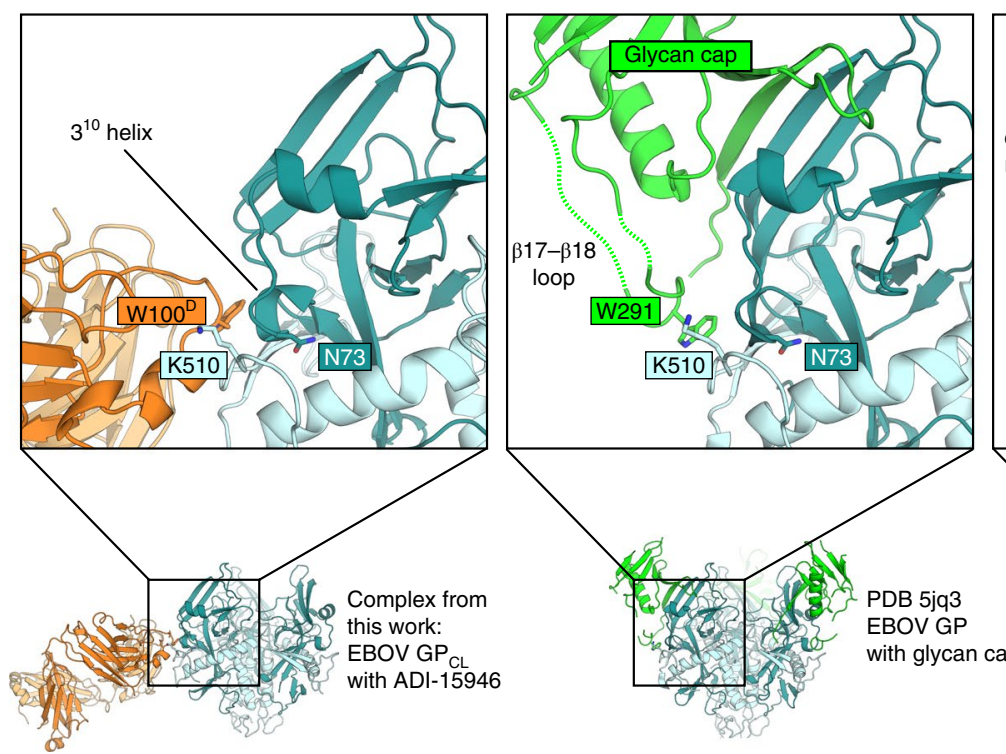

b

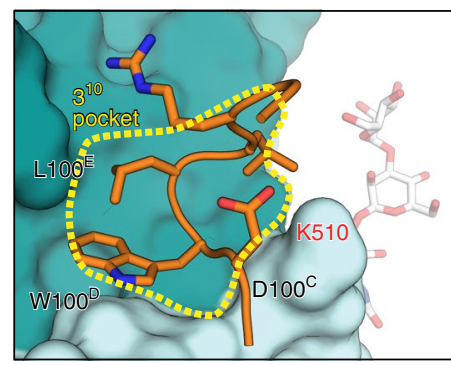

ADI-15946 CDRH3

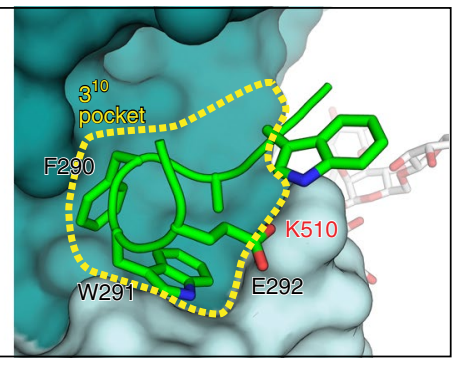

EBOV GP1 $\beta 17-\beta 18$ loop

d

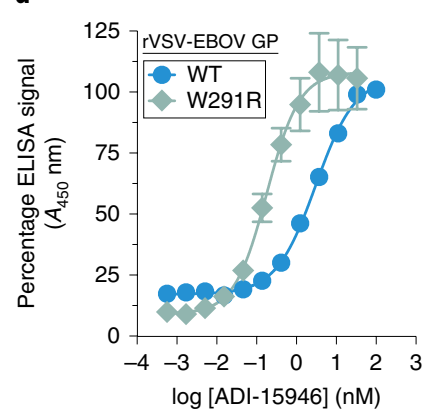

e

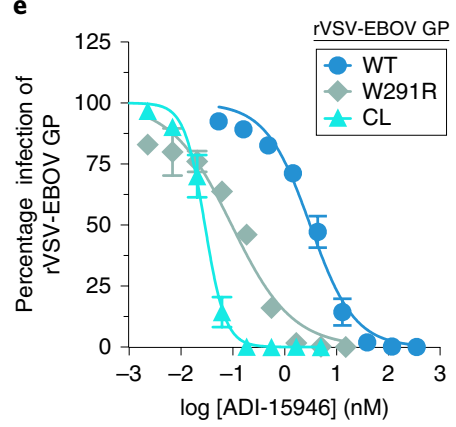

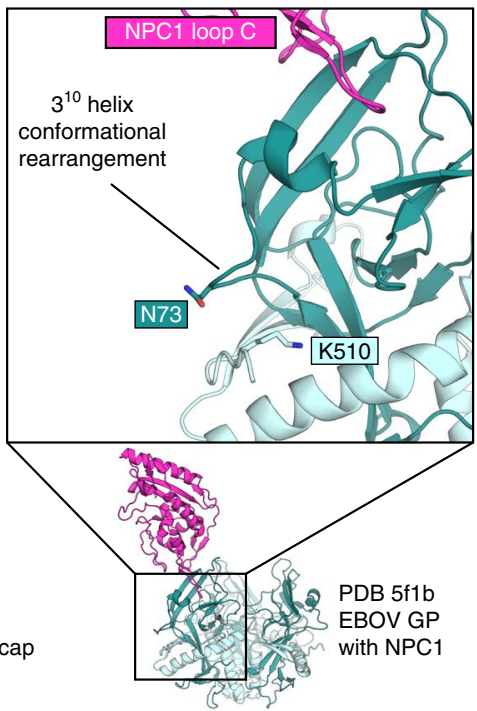

c
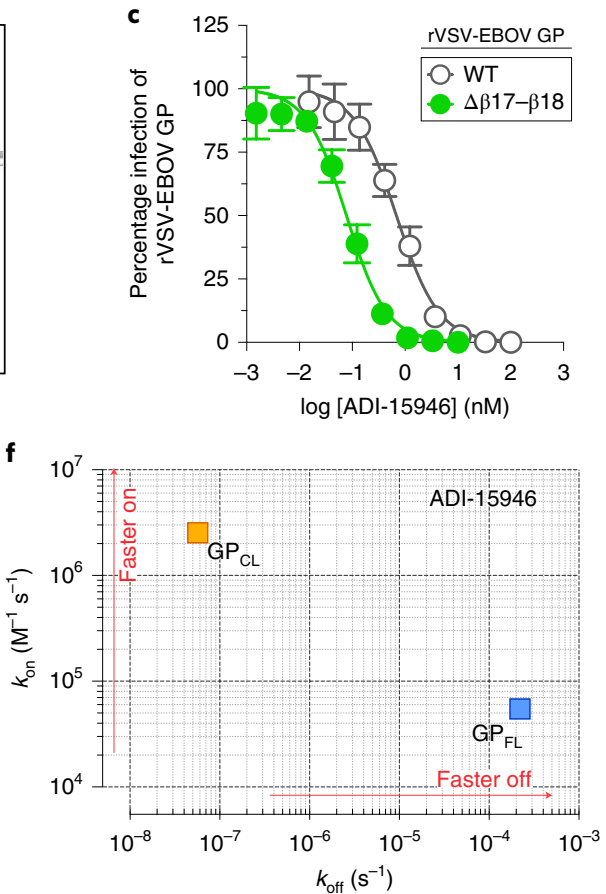

Fig. 3 | ADI-15946 binds a highly conserved epitope shielded by the mobile $\beta 17-\beta 18$ loop of the glycan cap. a, Structures show that ADI-15946 (orange, left) binds the hydrophobic $3_{10}$ pocket of GP (teal) that is usually occupied by the $\beta 17-\beta 18$ loop of the glycan cap (green, middle). Right, binding NPC1 loop C (magenta) to GP induces a conformational change in which the GP1 $3_{10}$ helix unwinds and asparagine 73 (N73) becomes solvent exposed while GP2 lysine 510 (K510) inserts into the cavity left behind after the unwinding of the $3_{10}$ helix. ADI-15946 may prevent these conformational changes from occurring by locking down the $3_{10}$ helix with residues that mimic those of the $\beta 17-\beta 18$ loop. $\mathbf{b}$, An enlarged view of the $3_{10}$ pocket shows that CDR H3 of ADI-15946 positions similar residues in similar orientations to that of the $\beta 17-\beta 18$ loop. c, Neutralization assay showing that ADI-15946 has enhanced neutralization of a GP construct lacking the $\beta 17-\beta 18$ loop compared to wild type (WT), probably due to increased access to the $3_{10}$ pocket. Data are mean \pm s.d., $n=6$ biologically independent samples. d, Binding assays showing that a point mutation in the $\beta 17-\beta 18$ loop (W291R) results in enhanced binding to rVSV-EBOV GP in an ELISA. Data are mean \pm s.d., $n=4$ biologically independent samples. e, Neutralization assays showing that the W291R mutation in the $\beta 17-\beta 18$ loop or its proteolytic removal $(\mathrm{CL})$ enhance the capacity of ADI-15946 to neutralize rVSV-EBOV GP. Data are mean \pm s.d., $n=6$ biologically independent samples. f, Kinetic binding studies by biolayer interferometry reveal enhanced association rate and slower dissociation rate of ADI-15946 to $\mathrm{GP}_{\mathrm{CL}}$ compared to uncleaved GP.

its footprint (Fig. $6 \mathrm{f}$ and Supplementary Note 2). There are only five nonconserved residues across all five ebolavirus GPs involved in the interface with ADI-15946, all located in GP2: N506, N514, H516, L547 and H549. These are predominantly contacted by CDR L2 and FRL3 (Fig. 6f). However, CDR H3 contacts one key residue in EBOV GP (Asn 506) that is not conserved in SUDV GP (Arg 506).
Using this information, we separately mutated nearby residues in ADI-15946 CDR H3, R100 and $\mathrm{Y}_{100^{\mathrm{A}}}$ to alanine to reduce steric and charge clashes with SUDV R506. The ADI-15946 mutant $\mathrm{Y} 100^{\mathrm{A}} \mathrm{A}$ showed decreased binding to both SUDV and BDBV GP compared to wild-type ADI-15946 (Supplementary Fig. 7). However, the mAb variant containing R100A, called 46M1, showed 

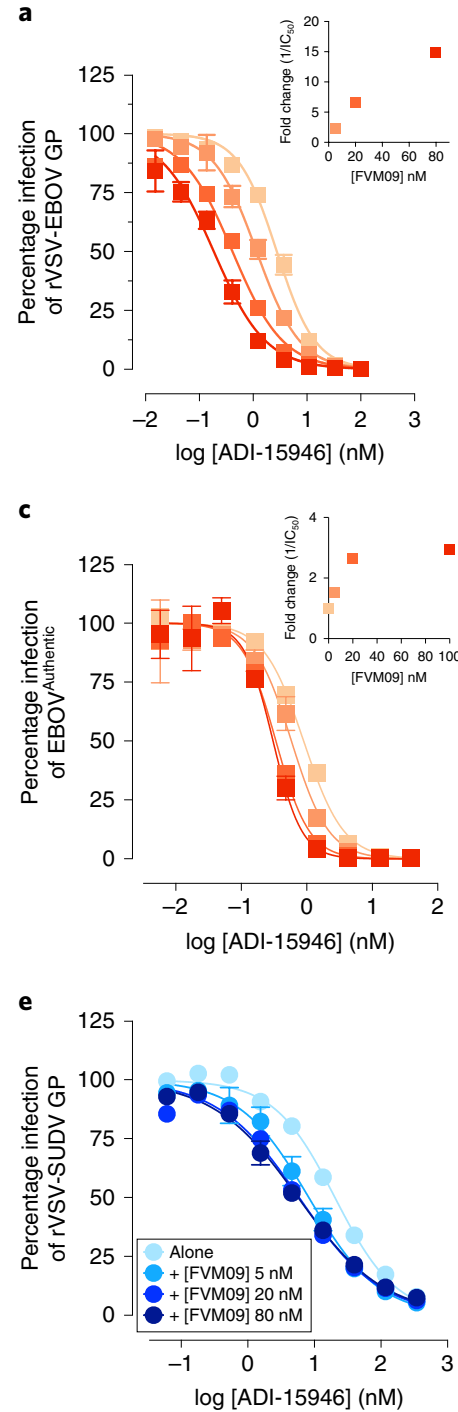

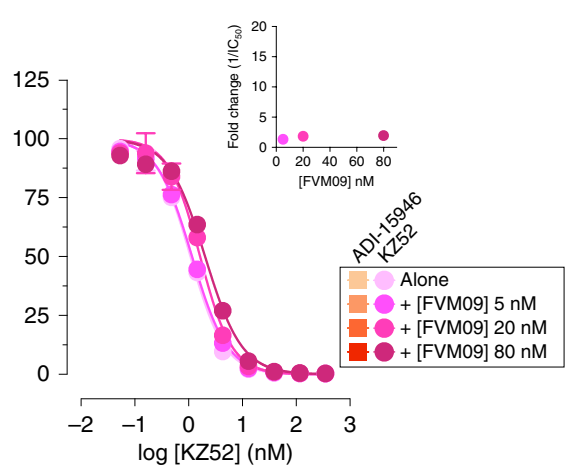

b

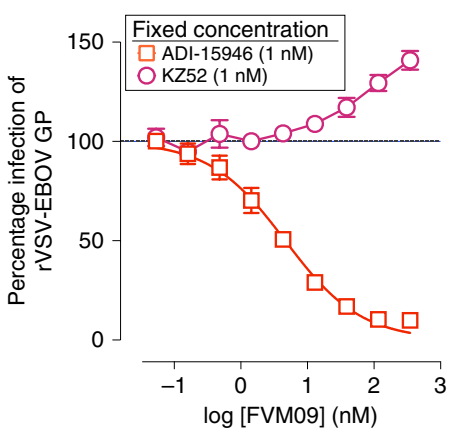

d

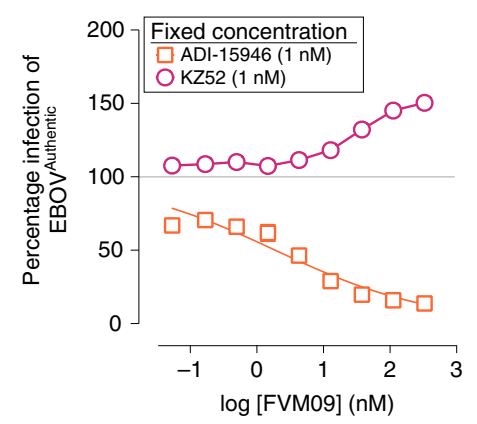

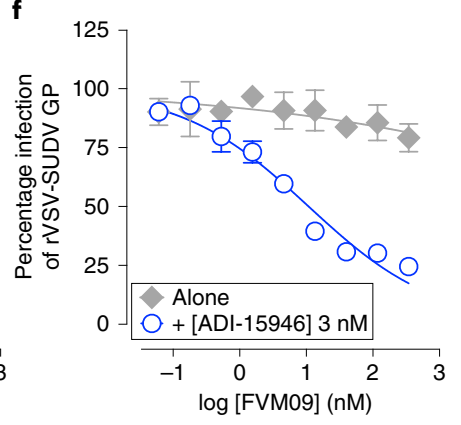

g

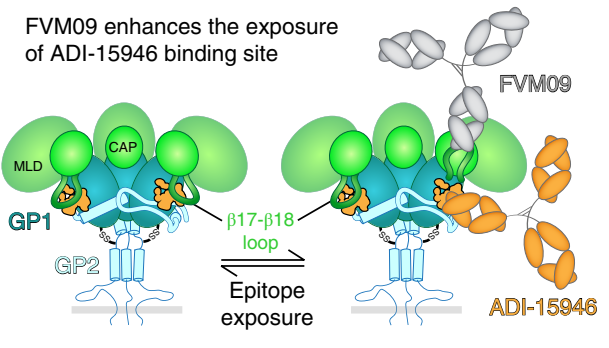

Fig. 4 | mAb FVM09 potentiates ADI-15946 neutralization of EBOV GP in a dose-dependent manner. a, Infection assays showing that addition of increasing concentrations of ADI-15946 (left), but not KZ52 (right), to fixed concentrations of FVMO9 (5, 20 or 80 nM) promotes rVSV-EBOV GP neutralization. The reciprocal fold change in neutralization half-maximal inhibitory concentration $\left(\mathrm{IC}_{50}\right.$ ) is shown (inset). $\mathbf{b}$, Infection assays showing that addition of increasing concentrations of FVMO9 to a fixed, subneutralizing concentration of ADI-15946 enhances rVSV-EBOV GP neutralization. The same experiment against KZ52 shows inhibition of neutralization as the concentration of FVM09 is increased. $\mathbf{c}-\mathbf{f}$, Infection assays as in $\mathbf{a}$, $\mathbf{b}$, with authentic EBOV (c,d) showing similar trends; this trend is present but less pronounced with rVSV-SUDV GP (e,f). Data in a-f are mean $\pm s . d ., n=6$ biologically independent samples. $\mathbf{g}$, A cartoon showing the proposed relationship between FVM09 binding and subsequent exposure of the ADI-15946 binding site.

increased binding to SUDV GP ectodomain by ELISA and slightly improved neutralization of authentic virus compared to the parental antibody (Fig. 6b-e and Supplementary Fig. 7).

Structural alignment of additional Fab-GP complexes enabled comparison of the binding features of the ADI-15946 paratope with those of EBOV-monospecific mAbs KZ52, c2G4 and c4G7, which have overlapping epitopes with that of ADI-15946 (Supplementary Fig. 5 and Supplementary Note 1$)^{6,27}$. We observed that the heavy chain paratope of $c 4 \mathrm{G} 7$ places a tyrosine residue in a similar position and orientation to that of the ADI-15946 LC residue F67 and another tyrosine residue proximal to ADI-15946 LC residue S65. We then attempted to mimic this double tyrosine motif by incorporation of light chain S65Y and F67Y mutations into ADI-15946. The construct bearing heavy chain R100A and light chain S65Y is called variant $46 \mathrm{M} 2$, and $\mathrm{ADI}-15946$ bearing the R100A/S65Y pair plus LC substitution F67Y is called variant 46M3 (Fig. 6a). The 46M2 and 46M3 antibodies showed enhanced binding of SUDV, while maintaining parental binding to EBOV and BDBV GPs (Fig. 6b).
The $46 \mathrm{M} 2$ and $46 \mathrm{M} 3$ antibodies also exhibited a 16- to 33 -fold improvement in their capacity to neutralize SUDV GP-bearing rVSV and authentic SUDV respectively, while retaining neutralization of EBOV and BDBV (Fig. 6c-e and Supplementary Fig. 7). Slight variations were observed in the neutralization profiles of the affinity variants versus the parent ADI-15946: we observed a roughly two-fold increase in neutralization for the affinity variants against rVSV-EBOV GP compared to wild-type ADI-15946 and a two-fold decrease in neutralization for the affinity variants against authentic EBOV compared to wild-type ADI-15946 (Fig. 6d,e and Supplementary Tables 1 and 3). Further, the variants and the parental antibody similarly recognize the conserved $3_{10}$ pocket, as all are equally enhanced by $\beta 17-\beta 18$ loop deletion and, such as the wildtype ADI-15946, 46M3 neutralization is enhanced in the presence of FVM09 (Fig. 6g and Supplementary Fig. 8). We show that relatively few mutations introduced in the $46 \mathrm{M} 2$ and $46 \mathrm{M} 3$ variants resulted in improved binding and neutralization of SUDV and confirmed the importance of the LC FR3 region to the binding interface. 


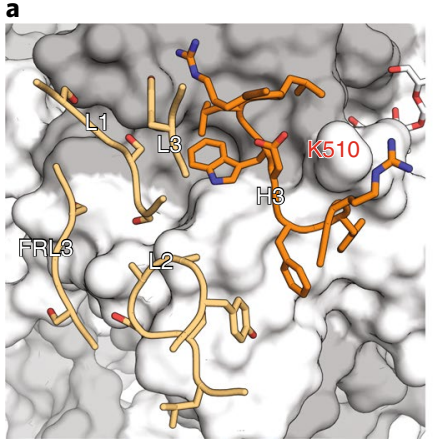

ADI-15946 IGL

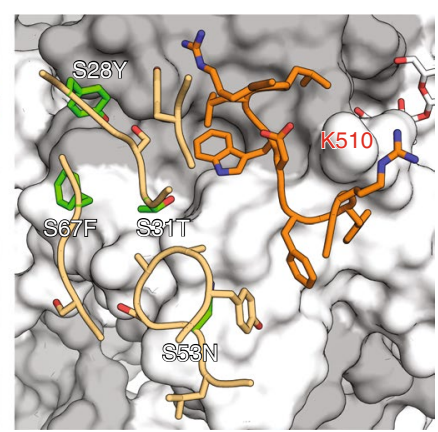

ADI-15946 WT b
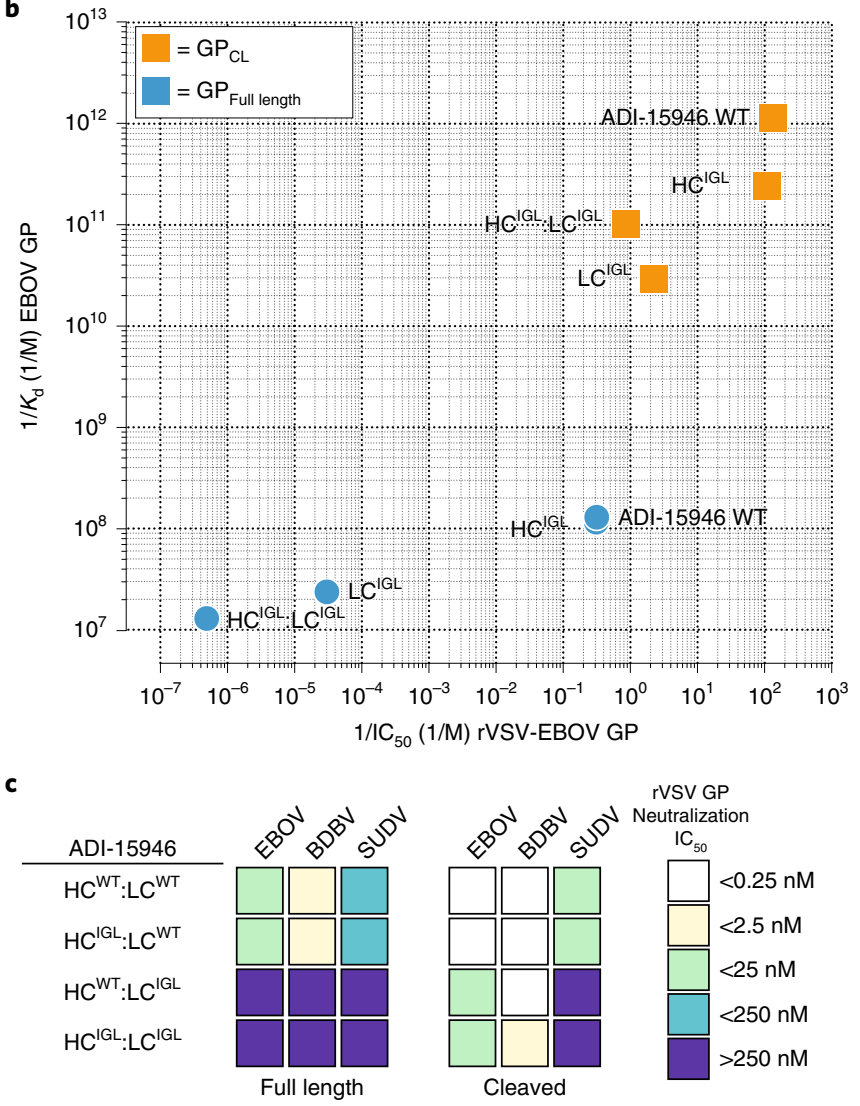

Fig. 5 | Genesis of ADI-15946. a, Models showing differences between the ADI-15946 IGL sequence (left) and the mature antibody (wild type, WT, right). GP1 is shown in gray and GP2 in white. $\mathbf{b}$, Comparison of the apparent equilibrium dissociation constant $\left(1 / K_{d}\right.$ app; higher value is tighter binding) for binding of ADI-15946 variants (WT, IGL and WT:IGL chimeras) to $\mathrm{GP}_{\mathrm{CL}}$ to their capacity to neutralize rVSV-EBOV GP infection $\left(1 / \mathrm{IC}_{50}\right.$; higher value is more potent neutralization). c, Heat maps for neutralization of rVSVs bearing ebolavirus GP and $\mathrm{GP}_{\mathrm{CL}}$ proteins by the indicated ADI15946 variants.

\section{Discussion}

The crystal structure of ADI-15946 Fab in complex with EBOV $\mathrm{GP}_{\mathrm{CL}}$ and accompanying biochemistry and virology presented here illuminated antigenic features of GP that dictate access to a broadly conserved but previously unappreciated epitope shared by ebolaviruses. This site of vulnerability is shielded by the descending $\beta 17-\beta 18$ loop of the glycan cap, removal of which potentiates ADI-15946 activity. Our findings support a mechanism of action whereby ADI-15946 gains enhanced neutralizing activity against the endosomal, cleaved viral GP species generated by host proteases during entry. This feature allows ADI-15946 to neutralize both the extracellular and intracellular viral species. In contrast, other potent neutralizers that target the GP base, such as KZ52 and the ZMapp components c2G4 and c4G7, have been shown to lose their activity against $\mathrm{GP}_{\mathrm{CL}}$ and therefore have activity only against the extracellular virus $\mathrm{s}^{6,20,27}$. The unique mechanism of neutralization by ADI15946 may involve binding of ADI-15946 in the $3_{10}$ pocket, which in turn prevents rearrangement of GP1 residues $71-75$ on binding of $\mathrm{GP}_{\mathrm{CL}}$ to NPC1 by mimicking the hydrophobic interactions of the $\beta 17-\beta 18$ loop that packs into the pocket in $\mathrm{GP}_{\mathrm{FL}}$ (Fig. $\left.3 \mathrm{a}, \mathrm{b}\right)^{12}$. In addition, ADI-15946 may also inhibit conformational changes required for membrane fusion by anchoring to the GP1-GP2 interface across the IFL thereby preventing its unraveling from the GP1 core during fusion triggering.

We also uncovered potentially synergistic neutralization between ADI-15946 and FVM09, a non-neutralizing glycan cap-binding antibody. FVM09 was previously shown to enhance binding activity of another glycan cap $\mathrm{mAb}, \mathrm{m} 8 \mathrm{C} 4$, yet reduce binding of the basetargeting $\mathrm{mAb}$ KZ52-the latter being consistent with our own observations $^{30}$. As with ADI-15946, mutations of the $\beta 17-\beta 18$ loop also enhanced m8C4's binding and neutralizing activity in vitro ${ }^{30}$. The exact mechanism of FVM09 and m8C4 cooperation is difficult to discern without structural details of the binding interface between the two mAbs and GP. In the case of cooperativity between FVM09 and ADI-15946, however, we propose that FVM09 primarily plays a supporting role by exposing the $3_{10}$ pocket thereby enhancing ADI-15946 binding and neutralization. It is possible that co-administration of FVM09 and ADI-15946 would lead to enhancement in protective efficacy in vivo. However, given that FVM09 is a nonneutralizing and non-protective antibody, viral escape from ADI15946 neutralization would render the two antibody combination non-protective. Follow up studies will be required to fully evaluate the therapeutic usefulness of such antibody combinations.

Our analysis of the contributions of somatic hypermutation to the binding and neutralization properties of ADI-15946 indicated that only a limited number of amino acid substitutions in the germline antibody sequence were required for binding of this conserved GP site and that many of these mutations occurred in the framework region. Klein et al. show that somatic mutations in the immunoglobulin framework region of antibodies against HIV-1 enhance affinity by decreasing the dissociation rate and are generally necessary to achieve broad neutralization ${ }^{31}$. We found that, similar to bNAbs against HIV-1, ADI-15946 also required somatic maturation of the framework region, which bestowed the mAb with an improved dissociation rate and broad neutralizing activity of ebolaviruses ${ }^{31}$. Specifically, as a result of SHM, a serine at position 67 of ADI-15946 FRL3 was replaced with an aromatic phenylalanine sidechain that interacts with GP2 residue H516 (100\% conserved between EBOV, BDBV and SUDV). Interestingly, the non-pathogenic RESTV encodes a tyrosine at position 516 in GP2, which may play a role in the inability of ADI-15946 to neutralize RESTV due to steric hindrance with the antibody phenylalanine 67 (Supplementary Fig. 7e and Supplementary Note 2).

This structure provided the molecular details of the paratope required for rational engineering of the parent $\mathrm{mAb}$ to expand its activity against SUDV. We showed that structure-guided substitutions, two in the FRL3 and one in CDR H3, enhanced both binding and neutralization activity against SUDV without loss of activity against EBOV or BDBV. However, follow-up work will be required to evaluate additional $\mathrm{mAb}$ variants and their biophysical properties to ensure favorable in vivo pharmacokinetics and protective efficacy against SUDV since, unlike natural antibodies, mAbs generated through in vitro engineering have not undergone immune tolerance selection. Our work also details structural features of GP recognition by a broadly active anti-ebolavirus antibody and suggests specific strategies such as the use of $\mathrm{GP}_{\mathrm{CL}}$ (lacking the glycan cap) or GP variants with $\beta 17-\beta 18$ loop deletion to enable targeting 


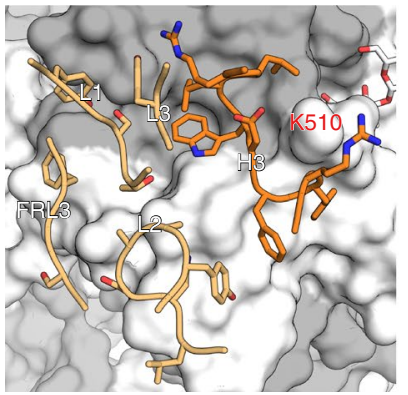

ADI-15946 WT

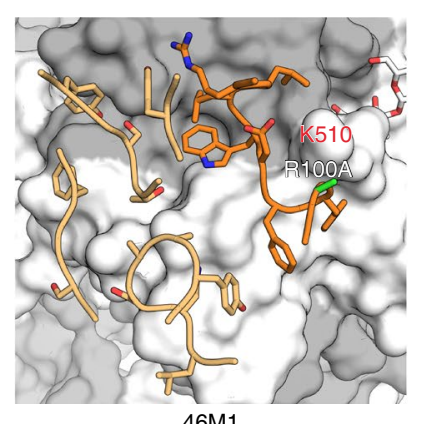

46M1

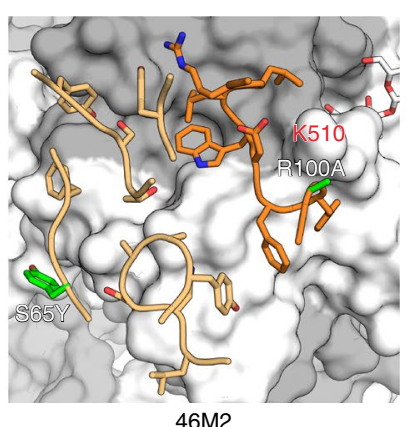

$46 \mathrm{M} 2$

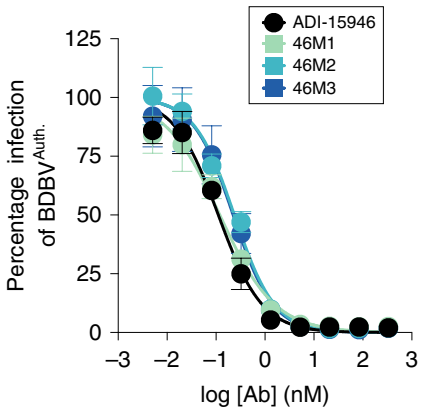

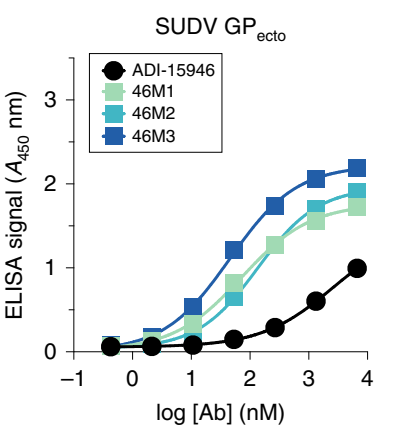

BDBV $\mathrm{GP}_{\text {ecto }}$
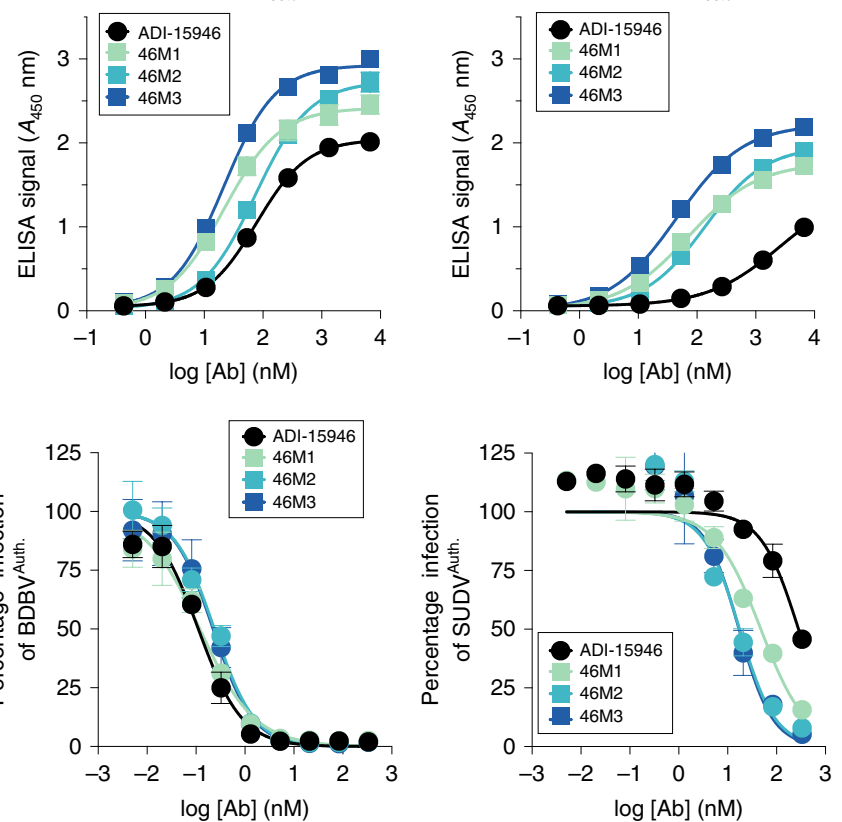

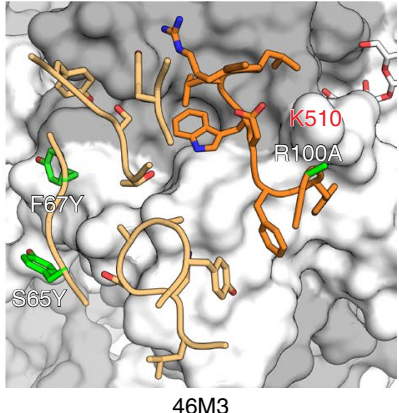

d

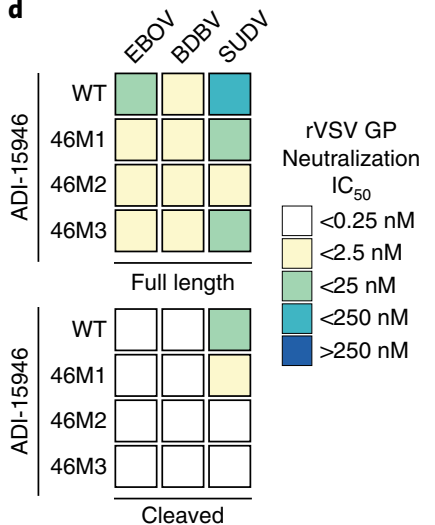

Authentic ebolavirus

e

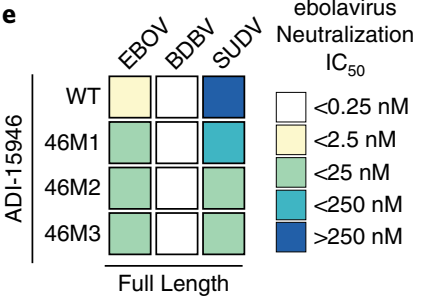

f

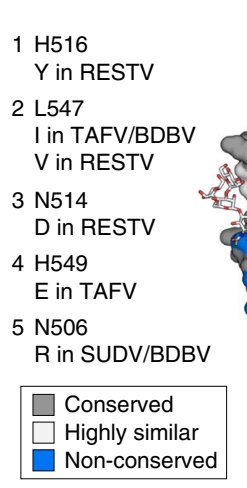

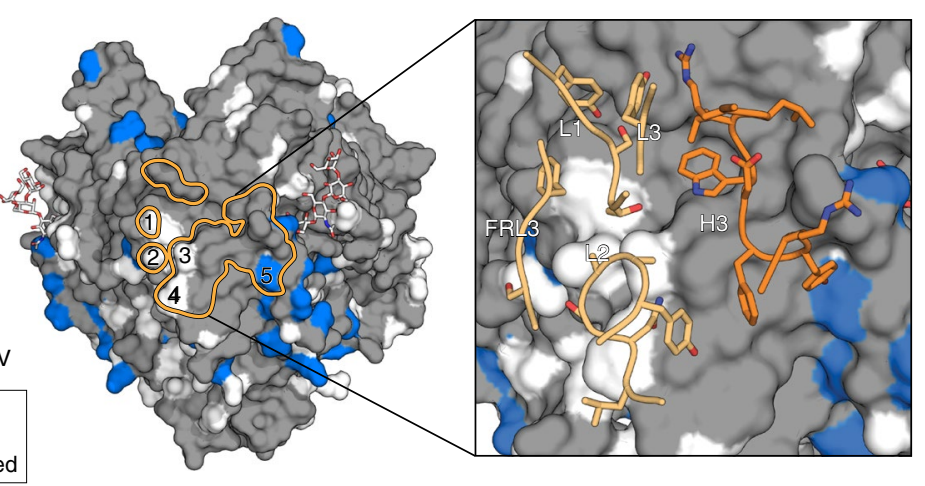

g

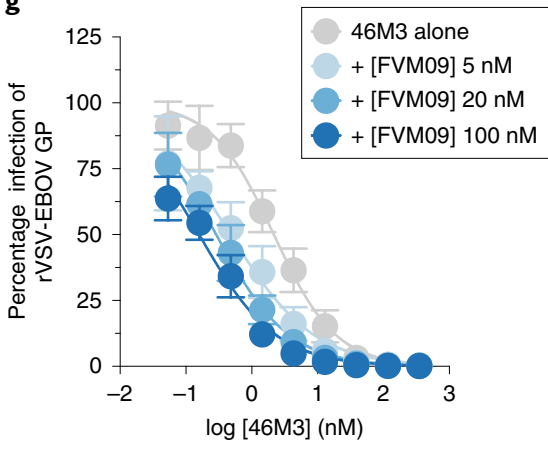

Fig. 6 | Structure-guided affinity maturation of ADI-15946. a, Molecular models showing the locations of mutations in ADI-15946 variants 46M1, $46 \mathrm{M} 2$ and $46 \mathrm{M} 3$ in relation to the surface of GP. CDRs are illustrated in dark orange for the heavy chain and light orange for the light chain, respectively; engineered side chains that differ from wild type are colored in green. GP1 and GP2 are shown as a gray and a white surface, respectively. b, Binding assays of recombinant EBOV, BDBV and SUDV GP ectodomains by the indicated ADI-15946 variants determined by ELISA. Data are mean \pm s.d., $n=3$ biologically independent samples. c, Neutralization assays of authentic EBOV, BDBV and SUDV by the indicated ADI-15946 variants. Data are mean \pm s.d., $n=6$ biologically independent samples. d,e, Heat maps for neutralization potency (IC s0 ) of each ADI-15946 variant against $r$ VSVs (d) and authentic filoviruses (e). In d, neutralization of rVSVs bearing full-length GPs and cleaved GPs is shown on the top and bottom, respectively. f. Molecular surface of EBOV GP ${ }_{C L}$ with the ADI-15946 footprint outlined in orange. Differences at five sites are listed on the left. The panel on the right shows which CDRs are in proximity to these nonconserved sites. TAFV, Taï Forest virus. g, Neutralization assays of rVSV-EBOV GP by $46 M 3$ in the presence of increasing concentrations of FVM09 (0-100 nM). Increasing amounts of FVM09 improved 46M3 neutralization. Data are mean \pm s.d., $n=6$ biologically independent samples. 
of the highly conserved site in the design of vaccines and immunotherapeutics. Like ADI-15946, the pan-ebolavirus mAb ADI15878 also binds into a cryptic pocket at a distinct site in the GP base, that is typically occupied by the N-terminal tail of GP2 when not bound by the $\mathrm{mAb}^{21}$. Future ebolavirus vaccine designs may strive to enhance the exposure of both of these vulnerable pockets on a single antigen by developing an immunogen that combines $\beta 17-\beta 18$ loop deletion with a truncated GP2 N terminus. Such immunogens may help elicit bNAbs such as ADI-15946 at a higher frequency while minimizing potential for viral escape by eliciting bNAbs towards multiple broadly conserved sites, both located proximal to the functionally critical region of the GP fusion loop. A broadly reactive vaccine would afford increased preparedness against ebolavirus disease regardless of the specific virus responsible for an outbreak.

\section{Online content}

Any methods, additional references, Nature Research reporting summaries, source data, statements of data availability and associated accession codes are available at https://doi.org/10.1038/ s41594-019-0191-4.

Received: 23 November 2018; Accepted: 23 January 2019; Published online: 4 March 2019

\section{References}

1. Qiu, X. et al. Reversion of advanced Ebola virus disease in nonhuman primates with ZMapp. Nature 514, 47-53 (2014).

2. Corti, D. et al. Protective monotherapy against lethal Ebola virus infection by a potently neutralizing antibody. Science 351, 1339-1342 (2016).

3. Pascal, K. E. et al. Development of clinical-stage human monoclonal antibodies that treat advanced Ebola virus disease in non-human primates. J. Infect. Dis. 218, S612-S626 (2018).

4. Cox, E. et al. Notes for the Record: Consultation on Monitored Emergency Use of Unregistered and Investigational Interventions for Ebola Virus Disease http:// www.who.int/emergencies/ebola/MEURI-Ebola.pdf?ua=1 (World Health Organization, 2018).

5. Sivapalasingam, S. et al. Safety, pharmacokinetics, and immunogenicity of a co-formulated cocktail of three human monoclonal antibodies targeting Ebola virus glycoprotein in healthy adults: a randomised, first-in-human phase 1 study. Lancet. Infect. Dis. 18, 884-893 (2018).

6. Lee, J. E. et al. Structure of the Ebola virus glycoprotein bound to an antibody from a human survivor. Nature 454, 177-182 (2008).

7. Volchkov, V. E., Feldmann, H., Volchkova, V. A. \& Klenk, H. D. Processing of the Ebola virus glycoprotein by the proprotein convertase furin. Proc. Natl Acad. Sci. USA 95, 5762-5767 (1998).

8. Gregory, S. M. et al. Structure and function of the complete internal fusion loop from Ebola virus glycoprotein 2. Proc. Natl Acad. Sci. USA 108, 11211-11216 (2011)

9. Lee, J. et al. Structure of the Ebola virus envelope protein MPER/TM domain and its interaction with the fusion loop explains their fusion activity. Proc. Natl Acad. Sci. USA 114, E7987-E7996 (2017).

10. Weissenhorn, W., Carfí, A., Lee, K. H., Skehel, J. J. \& Wiley, D. C. Crystal structure of the Ebola virus membrane fusion subunit, GP2, from the envelope glycoprotein ectodomain. Mol. Cell 2, 605-616 (1998).

11. Malashkevich, V. N. et al. Core structure of the envelope glycoprotein GP2 from Ebola virus at 1.9 A resolution. Proc. Natl Acad. Sci. USA 96, 2662-2667 (1999).

12. Wang, H. et al. Ebola viral glycoprotein bound to its endosomal receptor Niemann-Pick C1. Cell 164, 258-268 (2016).

13. Schornberg, K. et al. Role of endosomal cathepsins in entry mediated by the Ebola virus glycoprotein. J. Virol. 80, 4174-4178 (2006).

14. Chandran, K., Sullivan, N. J., Felbor, U., Whelan, S. P. \& Cunningham, J. M. Endosomal proteolysis of the Ebola virus glycoprotein is necessary for infection. Science 308, 1643-1645 (2005).

15. Hood, C. L. et al. Biochemical and structural characterization of cathepsin L-processed Ebola virus glycoprotein: implications for viral entry and immunogenicity. J. Virol. 84, 2972-2982 (2010).

16. Bornholdt, Z. A. et al. Host-primed Ebola virus GP exposes a hydrophobic NPC1 receptor-binding pocket, revealing a target for broadly neutralizing antibodies. mBio 7, e02154-15 (2016).
17. Côté, M. et al. Small molecule inhibitors reveal Niemann-Pick C1 is essential for Ebola virus infection. Nature 477, 344-348 (2011).

18. Carette, J. E. et al. Ebola virus entry requires the cholesterol transporter Niemann-Pick C1. Nature 477, 340-343 (2011).

19. Miller, E. H. et al. Ebola virus entry requires the host-programmed recognition of an intracellular receptor. EMBO J. 31, 1947-1960 (2012).

20. Wec, A. Z. et al. Antibodies from a human survivor define sites of vulnerability for broad protection against ebolaviruses. Cell 169, 878-890 (2017).

21. West, B. R. et al. Structural basis of pan-ebolavirus neutralization by a human antibody against a conserved, yet cryptic epitope. mBio 9, e01674-18 (2018).

22. Saphire, E. O. et al. Systematic analysis of monoclonal antibodies against Ebola virus GP defines features that contribute to protection. Cell 174, 938-952 (2018).

23. Furuyama, W. et al. Discovery of an antibody for pan-ebolavirus therapy. Sci. Rep. 6, 20514 (2016)

24. Milligan, J. C. et al. Structural characterization of pan-ebolavirus antibody 6D6 targeting the fusion peptide of the surface glycoprotein. J. Infect. Dis. 219, 415-419 (2019).

25. Bornholdt, Z. A. et al. Isolation of potent neutralizing antibodies from a survivor of the 2014 Ebola virus outbreak. Science 351, 1078-1083 (2016)

26. Zhao, $X$. et al. Immunization-elicited broadly protective antibody reveals ebolavirus fusion loop as a site of vulnerability. Cell 169, 891-904 (2017).

27. Pallesen, J. et al. Structures of Ebola virus GP and sGP in complex with therapeutic antibodies. Nat Microbiol 1, 16128 (2016).

28. Zhao, Y. et al. Toremifene interacts with and destabilizes the Ebola virus glycoprotein. Nature 535, 169-172 (2016).

29. Keck, Z. et al. Macaque monoclonal antibodies targeting novel conserved epitopes within filovirus glycoprotein. J. Virol. 90, 279-291 (2015).

30. Howell, K. A. et al. Cooperativity enables non-neutralizing antibodies to neutralize Ebola virus. Cell Rep. 19, 413-424 (2017).

31. Klein, F. et al. Somatic mutations of the immunoglobulin framework are generally required for broad and potent HIV-1 neutralization. Cell 153, 126-138 (2013).

\section{Acknowledgements}

$\mathrm{X}$-ray diffraction data were collected at the Stanford Synchrotron Radiation Lightsource (SSRL), SLAC National Accelerator Laboratory. SSRL is supported by the U.S. Department of Energy, Office of Science, Office of Basic Energy Sciences under Contract No. DE-AC02-76SF00515. The SSRL Structural Molecular Biology Program is supported by the DOE Office of Biological and Environmental Research, and by the National Institutes of Health, National Institute of General Medical Sciences (including P41GM103393). Opinions, conclusions, interpretations, and recommendations are those of the authors and are not necessarily endorsed by the U.S. Army. The mention of trade names or commercial products does not constitute endorsement or recommendation for use by the Department of the Army or the Department of Defense. We acknowledge National Institutes of Health grants no. U19 AI109762 (E.O.S., K.C., J.M.D.), no. R01 AI132256 (K.C.), no. U19 AI109711 (A.B.), no. R01 AI132204 (E.O.S., M.J.A.) and no. R01 AI126587 (M.J.A.), the Defense Threat Reduction Agency HDTRA1-131-0034 (A.B.) and the Viral Hemorrhagic Fever Immunotherapeutic Consortium for support. This is manuscript number 29630 from The Scripps Research Institute.

\section{Author contributions}

B.R.W., A.Z.W., C.L.M., M.L.F., P.A.I., K.H., A.S.W., R.M.J., A.S.H., S.H., E.G., K.A.H. and S.K. carried out the research. B.R.W., A.Z.W., K.C. and E.O.S. designed the study. M.J.A. contributed materials. L.M.W., J.M.D., A.B., K.C. and E.O.S. supervised the research. B.R.W., A.Z.W., K.C. and E.O.S. drafted the manuscript. B.R.W., A.Z.W., K.C. and E.O.S edited the manuscript. All authors analyzed data and commented on the drafts.

\section{Competing interests}

M.J.A. has stock in Integrated Biotherapeutics, a company developing antibody therapeutics for ebolavirus disease. A.Z.W., E.G. and L.M.W. are employees and equity holders of Adimab. K.C. and J.M.D. are members of the Scientific Advisory Board of Integrum Scientific, LLC.

\section{Additional information}

Supplementary information is available for this paper at https://doi.org/10.1038/ s41594-019-0191-4.

Reprints and permissions information is available at www.nature.com/reprints. Correspondence and requests for materials should be addressed to K.C. or E.O.S.

Publisher's note: Springer Nature remains neutral with regard to jurisdictional claims in published maps and institutional affiliations.

(C) The Author(s), under exclusive licence to Springer Nature America, Inc. 2019 


\section{Methods}

Cloning. Mutants were introduced into parental ADI-15946 by site-directed mutagenesis using QuickChange (Agilent), with all mutations confirmed by sequencing (Eton)

Protein expression and purification. Expression and purification of EBOV GP was carried out as described previously ${ }^{16}$. Briefly, ebolavirus GP (lacking the mucin domain residues 312-462) was produced by stable expression in Drosophila melanogaster S2 cells. Effectene (Qiagen) was used to transfect S2 cells with a modified pMT-puro vector plasmid containing the GP gene of interest, followed by stable selection of transfected cells with $6 \mu \mathrm{g} \mathrm{ml}^{-1}$ puromycin. Cells were cultured at $27^{\circ} \mathrm{C}$ in complete Schneider's medium for selection and then adapted to Insect Xpress medium (Lonza) for large-scale expression in 2-L Erlenmeyer flasks. Secreted GP ectodomain expression was induced with $0.5 \mathrm{mM} \mathrm{CuSO}_{4}$, and supernatant collected after 4 days. Ebolavirus GP was engineered with a double Strep-tag at the $C$ terminus to facilitate purification using Strep-Tactin resin (2-1201-010) (Qiagen) and then further purified by Superdex 200 (GE) size exclusion chromatography (SEC) in $10 \mathrm{mM}$ Tris-buffered saline (Tris-HCl, pH 7.5, $150 \mathrm{mM} \mathrm{NaCl}(\mathrm{TBS}))$. EBOV GP $\mathrm{CL}$ was produced by incubation of $1 \mathrm{mg} \mathrm{GP}$ with $0.02 \mathrm{mg}$ thermolysin overnight at room temperature in TBS containing $1 \mathrm{mM}$ $\mathrm{CaCl}_{2}$ and purified using Superdex 200 SEC.

ADI-15946 Fab used for crystallization experiments was cloned into a modified pMT-puro vector with a heavy chain C-terminal Strep-tag, and then expressed and purified according to the protocol for $\mathrm{GP}_{\mathrm{CL}}$ with the exception that SEC was performed with a Superdex 75 column $(\mathrm{GE})^{16}$.

ADI-15946 IgG used for ELISA and neutralization assays were produced in ExpiCHO cells (ThermoFisher Scientific) and purified via Protein A chromatography according to the standard ThermoFisher ExpiCHO protocol for a $25 \mathrm{ml}$ culture volume. Cells were pelleted 8 days post transfection by centrifugation for $30 \mathrm{~min}$ at $3,000 \mathrm{~g}$ and supernatant was collected for purification of soluble ADI15946 IgG. Supernatant was flowed over $2 \mathrm{ml}$ of Pierce Protein A Plus Agarose resin (ThermoFisher Scientific). Column was washed with five column volumes of DPBS (Gibco) and then IgG was eluted with DPBS supplemented with $25 \mathrm{mM}$ glycine at $\mathrm{pH} 2.2$.

Crystallography and structure determination. Trimeric EBOV $\mathrm{GP}_{\mathrm{CL}}$ was in complex with ADI-15946 Fab fragments, and the resulting complex was then purified via SEC. The purified EBOV $\mathrm{GP}_{\mathrm{CL}}-\mathrm{ADI}-15946$ Fab complex was concentrated to $4.2 \mathrm{mg} \mathrm{ml}^{-1}$ in TBS. The crystal drops consisted of a 1:1 ratio of protein/well solution. Crystals grew over the course of 1 month in $0.2 \mathrm{M}$ sodium citrate tribasic dihydrate $\mathrm{pH} 8.2$ and $20 \%$ polyethylene glycol 3350 . Crystals were cryoprotected with $20 \%$ glycerol and flash frozen in liquid nitrogen for storage and shipping. Diffraction data was collected remotely on SSRL beamline 12-2 on a pilatus $6 \mathrm{M}$ detector ${ }^{32-35}$. Data was processed using $\mathrm{XDS}^{36,37}$, and the structure was determined using molecular replacement with PHASER ${ }^{38}$, in the CCP4 suite $^{39}$, using the structure of EBOV GP $\mathrm{CL}$ (PDB 5HJ3) as an initial search model ${ }^{16}$. Iterative rounds of model building were performed using $\operatorname{Coot}^{40}$, and each round was refined with Phenix ${ }^{41}$. Five percent of the data was set aside before refinement for the $R_{\text {free }}$ calculations for each data set ${ }^{42}$. The statistics and stereochemistry of the crystal structure were checked using the MolProbity server ${ }^{42,43}$. Structural figures were rendered using Open Source PyMOL (PyMOL Molecular Graphics System, v.1.7.0.0; Schrödinger, LLC).

Structural alignment and visualization of ebolavirus glycoproteins. Alignment was performed using clustalomega on uniprot with the following protein sequences: Zaire ebolavirus: Q05320, Bundibugyo ebolavirus: B8XCN0, Sudan ebolavirus: Q66814, Taï Forest ebolavirus: Q66810, Reston ebolavirus: Q66799. Sequence conservation was numbered according to EBOV GP and visualized using the Espript server (http://espript.ibcp.fr) and colored according to the percent equivalent scoring function with a cutoff of $70 \%$ (ref. ${ }^{44}$ ).

Determination of inferred germline progenitors. ADI-15946 heavy and light chain sequences were aligned to their nearest predicted germline progenitors using IMGT. Amino acid substitutions in the variable region of the heavy and light chain outside of CDR H3 and CDR L3 of the mature antibody were reverted to germline-encoded amino acids. Germline-reverted sequences of variable heavy and light chain domains were then ordered as gBlocks with overhangs to allow for homologous recombination into Saccharomyces cervisiae.

Anti-GP mAb ELISAs. High-binding 96-well ELISA plates (Corning) were coated with $50 \mu \mathrm{l} \mathrm{GP}$ antigens in phosphate-buffered saline (PBS) at $4 \mu \mathrm{g} \mathrm{ml}^{-1}$, and allowed to bind for $1 \mathrm{~h}$ at room temperature. After washing, the wells were blocked with PBS containing $3 \%$ bovine serum albumin (PBSA) for $1 \mathrm{~h}$ at room temperature, followed by washing then incubation with ADI-15946 or one of its mutant derivatives in serial dilutions of PBS. A horseradish-peroxidase conjugated antihuman secondary antibody (Santa Cruz Biotechnology) was added and allowed to bind for $1 \mathrm{~h}$ at room temperature and then subsequently detected by ultra-TMB $\left(3,3^{\prime}, 5,5^{\prime}\right.$-tetramethylbenzidine) substrate (ThermoFisher Scientific). Optical density was measured at $450 \mathrm{~nm}$, and absorbance readings were subjected to a nonlinear regression analysis (GraphPad Prism software) to generate binding curves and calculate a half-maximum effective concentration value. ELISA assays were performed in triplicate, across seven five-fold dilutions, beginning at $1,000 \mathrm{ng} \mathrm{ml}^{-1}$.

Biolayer interferometry assays. The Octet Red system (FortéBio, Pall) was used to determine the binding properties of different IgGs to various forms of EBOV GP. Anti-human Fc (AHC) capture sensors (ForteBio) were used for initial mAb loading at $25 \mathrm{mg} \mathrm{ml}^{-1}$ in $1 \times$ kinetics buffer (PBS supplemented with $0.002 \%$ Tween-20 and $1 \mathrm{mg} \mathrm{ml}^{-1}$ of BSA). Binding to GP was carried out across two-fold serial dilutions of EBOV GP $\Delta \mathrm{TM}$ or $\mathrm{GP}_{\mathrm{CL}}$. The baseline and dissociation steps were carried out in the $1 \times$ kinetics buffer as per the instrument manufacturer's recommendations. Kinetic binding data in all cases are adequately and accurately described by a 1:1 binding model, but given the bivalent nature of the IgG (immobilized) and the trimeric state of GP (analyte), the association stoichiometry is likely to be more complex. Thus, $k_{\text {off }} / k_{\text {on }}$ probably reflects an ensemble of binding stoichiometries and, accordingly, we refer to this ratio as apparent $K_{\mathrm{d}}\left(K_{\mathrm{d}}^{\text {app }}\right)$ throughout.

rVSV neutralization assays. Neutralization of rVSV: recombinant VSV expressing both enhanced green fluorescent protein (EGFP) and recombinant surface GP (rVSV-EBOV GP) in place of VSV G have been previously described ${ }^{45-47}$. Vero cells were seeded at $6.0 \times 104$ cells per well and cultured overnight in Eagle's minimal essential medium (EMEM) supplemented with $10 \%$ fetal bovine serum (FBS) and 100 I.U. per $\mathrm{ml}$ of penicillin and $100 \mathrm{mg} \mathrm{ml}^{-1}$ streptomycin at $37^{\circ} \mathrm{C}$ and $5 \% \mathrm{CO}_{2}$. The next day, virus was incubated with serial three-fold antibody dilutions beginning at $350 \mathrm{nM}\left(\sim 50 \mu \mathrm{g} \mathrm{ml}^{-1}\right)$ in serum free DMEM for $1 \mathrm{~h}$ at room temperature before infecting Vero cell monolayers in 96-well plates. The virus was incubated with the cells in 50\% v/v DMEM supplemented with $2 \% \mathrm{FBS}$, 100 I.U. per $\mathrm{ml}$ of penicillin and $100 \mu \mathrm{g} \mathrm{ml}^{-1}$ streptomycin at $37^{\circ} \mathrm{C}$ and $5 \% \mathrm{CO}_{2}$ for 14-16h before the cells were fixed and the nuclei stained with Hoescht 33342. rVSV infectivity was measured by counting EGFP-positive cells in comparison to the total number of cells indicated by nuclear staining using a Cellinsight CX5 automated microscope and accompanying software (ThermoFisher Scientific).

Authentic virus neutralization assays. Neutralization at BSL-4 was tested against replication-competent infectious EGFP-expressing EBOV and chimeric $\mathrm{EBOV} / \mathrm{BDBV}-\mathrm{GP}$ and EBOV/SUDV-GP constructs (referred to as EBOV, BDBV and SUDV, respectively) in HTS format, as previously described ${ }^{48}$. The neutralization assays were performed using Vero-E6 cells obtained from ATCC and maintained in minimal essential medium (MEM) (ThermoFisher Scientific) supplemented by $10 \%$ FBS (HyClone) and $1 \%$ penicillin-streptomycin at $5 \% \mathrm{CO}_{2}$, $37^{\circ} \mathrm{C}$. Neutralization assays were performed in triplicate, across 12 four-fold dilutions, starting from $200 \mu \mathrm{g} \mathrm{ml}^{-1}$.

Authentic virus cooperativity assays. The authentic Ebola virus/H. sapiens- tc/ COD/1995/Kikwit-9510621 (EBOV/Kik-9510621; 'EBOV-Zaire 1995') ${ }^{49}$, was used in this study. Antibodies were diluted to indicated concentrations in culture media and incubated with EBOV for $1 \mathrm{~h}$. Vero cells were exposed to antibody/virus inoculum at an MOI of 0.2 plaque-forming unit per cell for $1 \mathrm{~h}$. Antibody/virus inoculum was then removed and fresh culture media was added. At $48 \mathrm{~h}$ post-infection, cells were fixed with formalin, and blocked with $1 \%$ bovine serum albumin. EBOV-infected cells and uninfected controls were incubated with EBOV GP-specific mAb KZ52 $2^{50}$. Cells were washed with PBS before incubation with goat anti-human IgG conjugated to Alexa 488. Cells were counterstained with Hoechst 33342 stain (Invitrogen), washed with PBS and stored at $4^{\circ} \mathrm{C}$. Infected cells were quantitated by fluorescence microscopy and automated image analysis. Images were acquired at 20 fields per well with a $\times 20$ objective lens on an Operetta high content device (Perkin Elmer). Operetta images were analyzed with a customized scheme built from image analysis functions available in Harmony software.

Reporting Summary. Further information on research design is available in the Nature Research Reporting Summary linked to this article.

\section{Data availability}

Coordinates and structure factors have been deposited in the Protein Data Bank under accession number 6MAM. Other data are available from corresponding author upon reasonable request.

\section{References}

32. Russi, S., Song, J., McPhillips, S. E. \& Cohen, A. E. The Stanford automated mounter: pushing the limits of sample exchange at the SSRL macromolecular crystallography beamlines. J. Appl. Crystallogr. 49, 622-626 (2016).

33. Cohen, A. E., Ellis, P. J., Miller, M. D., Deacon, A. M. \& Phizackerley, R. P. An automated system to mount cryo-cooled protein crystals on a synchrotron beam line, using compact sample cassettes and a small-scale robot. J. Appl. Crystallogr. 35, 720-726 (2002).

34. Soltis, S. M. et al. New paradigm for macromolecular crystallography experiments at SSRL: automated crystal screening and remote data collection. Acta Crystallogr. D 64, 1210-1221 (2008). 
35. McPhillips, T. M. et al. Blu-Ice and the distributed control system: software for data acquisition and instrument control at macromolecular crystallography beamlines. J. Synchrotron. Radiat. 9, 401-406 (2002).

36. Kabsch, W. Integration, scaling, space-group assignment and post-refinement. Acta Crystallogr. D 66, 133-144 (2010).

37. Kabsch, W. XDS. Acta Crystallogr. D 66, 125-132 (2010).

38. McCoy, A. J. et al. Phaser crystallographic software. J. Appl. Crystallogr. 40, 658-674 (2007).

39. Collaborative Computational Project, Number 4. The CCP4 suite: programs for protein crystallography. Acta Crystallogr. D 50, 760-763 (1994).

40. Emsley, P., Lohkamp, B., Scott, W. G. \& Cowtan, K. Features and development of Coot. Acta Crystallogr. D . 66, 486-501 (2010).

41. Adams, P. D. et al. PHENIX: a comprehensive Python-based system for macromolecular structure solution. Acta Crystallogr. D 66, (213-221 (2010).

42. Brünger, A. T. Free R value: a novel statistical quantity for assessing the accuracy of crystal structures. Nature 355, 472-475 (1992).

43. Chen, V. B. et al. MolProbity: all-atom structure validation for macromolecular crystallography. Acta Crystallogr. D 66, 12-21 (2010).
44. Robert, X. \& Gouet, P. Deciphering key features in protein structures with the new ENDscript server. Nucleic Acids Res. 42, W320-W324 (2014).

45. $\mathrm{Ng}, \mathrm{M}$. et al. Cell entry by a novel European filovirus requires host endosomal cysteine proteases and Niemann-Pick C1. Virology 468-470, 637-646 (2014)

46. Wong, A. C., Sandesara, R. G., Mulherkar, N., Whelan, S. P. \& Chandran, K. A forward genetic strategy reveals destabilizing mutations in the Ebolavirus glycoprotein that alter its protease dependence during cell entry. J. Virol. 84, 163-175 (2010).

47. Wec, A. Z. et al. A 'Trojan horse' bispecific-antibody strategy for broad protection against ebolaviruses. Science 354, 350-354 (2016).

48. Ilinykh, P. A. et al. Chimeric filoviruses for identification and characterization of monoclonal antibodies. J. Virol. 90, 3890-3901 (2016).

49. Jahrling, P. B. et al. Evaluation of immune globulin and recombinant interferon-alpha2b for treatment of experimental Ebola virus infections. J. Infect. Dis. 179, S224-S234 (1999).

50. Maruyama, T. et al. Ebola virus can be effectively neutralized by antibody produced in natural human infection. J. Virol. 73, 6024-6030 (1999). 


\section{Reporting Summary}

Nature Research wishes to improve the reproducibility of the work that we publish. This form provides structure for consistency and transparency in reporting. For further information on Nature Research policies, see Authors \& Referees and the Editorial Policy Checklist.

\section{Statistical parameters}

When statistical analyses are reported, confirm that the following items are present in the relevant location (e.g. figure legend, table legend, main text, or Methods section).

$\mathrm{n} / \mathrm{a} \mid$ Confirmed

\The exact sample size $(n)$ for each experimental group/condition, given as a discrete number and unit of measurement

$\square$ An indication of whether measurements were taken from distinct samples or whether the same sample was measured repeatedly

$\triangle$ The statistical test(s) used AND whether they are one- or two-sided

Х Only common tests should be described solely by name; describe more complex techniques in the Methods section.

$\bigotimes \square$ A description of all covariates tested

Х $\square$ A description of any assumptions or corrections, such as tests of normality and adjustment for multiple comparisons

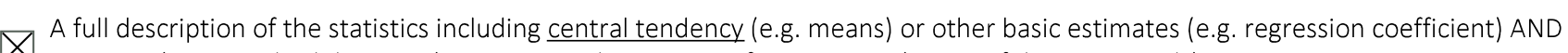

$\bigotimes$ variation (e.g. standard deviation) or associated estimates of uncertainty (e.g. confidence intervals)

W For null hypothesis testing, the test statistic (e.g. $F, t, r$ ) with confidence intervals, effect sizes, degrees of freedom and $P$ value noted

$\triangle$ Give $P$ values as exact values whenever suitable.

\ $\square$ For Bayesian analysis, information on the choice of priors and Markov chain Monte Carlo settings

Х $\square$ For hierarchical and complex designs, identification of the appropriate level for tests and full reporting of outcomes

Х $\square$ Estimates of effect sizes (e.g. Cohen's $d$, Pearson's $r$ ), indicating how they were calculated

Clearly defined error bars

State explicitly what error bars represent (e.g. SD, SE, Cl)

Our web collection on statistics for biologists may be useful.

\section{Software and code}

\section{Policy information about availability of computer code}

Data collection

Provide a description of all commercial, open source and custom code used to collect the data in this study, specifying the version used $O R$ state that no software was used.

Data analysis

Provide a description of all commercial, open source and custom code used to analyse the data in this study, specifying the version used

OR state that no software was used.

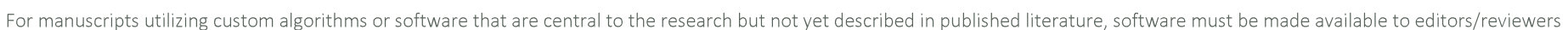

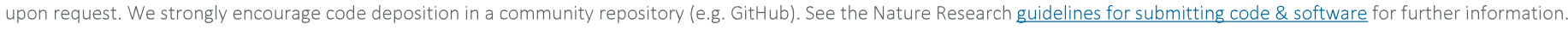

\section{Data}

Policy information about availability of data

All manuscripts must include a data availability statement. This statement should provide the following information, where applicable:

- Accession codes, unique identifiers, or web links for publicly available datasets

- A list of figures that have associated raw data

- A description of any restrictions on data availability

Structural data has been deposited in the PDB under accession code 6MAM. 


\section{Field-specific reporting}

Please select the best fit for your research. If you are not sure, read the appropriate sections before making your selection.

$\square$ Life sciences

Behavioural \& social sciences

Ecological, evolutionary \& environmental sciences

For a reference copy of the document with all sections, see nature.com/authors/policies/Reportingsummary-flat.pdf

\section{Life sciences study design}

All studies must disclose on these points even when the disclosure is negative.

Sample size Describe how sample size was determined, detailing any statistical methods used to predetermine sample size OR if no sample-size calculation was performed, describe how sample sizes were chosen and provide a rationale for why these sample sizes are sufficient.

Data exclusions Describe any data exclusions. If no data were excluded from the analyses, state so OR if data were excluded, describe the exclusions and the rationale behind them, indicating whether exclusion criteria were pre-established.

Replication Describe the measures taken to verify the reproducibility of the experimental findings. If all attempts at replication were successful, confirm this $O R$ if there are any findings that were not replicated or cannot be reproduced, note this and describe why.

Randomization Describe how samples/organisms/participants were allocated into experimental groups. If allocation was not random, describe how covariates were controlled OR if this is not relevant to your study, explain why.

Blinding

Describe whether the investigators were blinded to group allocation during data collection and/or analysis. If blinding was not possible, describe why OR explain why blinding was not relevant to your study.

\section{Behavioural \& social sciences study design}

All studies must disclose on these points even when the disclosure is negative.
Study description
Briefly describe the study type including whether data are quantitative, qualitative, or mixed-methods (e.g. qualitative cross-sectional, quantitative experimental, mixed-methods case study).
Research sample
State the research sample (e.g. Harvard university undergraduates, villagers in rural India) and provide relevant demographic information (e.g. age, sex) and indicate whether the sample is representative. Provide a rationale for the study sample chosen. For studies involving existing datasets, please describe the dataset and source.

Sampling strategy

Describe the sampling procedure (e.g. random, snowball, stratified, convenience). Describe the statistical methods that were used to predetermine sample size OR if no sample-size calculation was performed, describe how sample sizes were chosen and provide a rationale for why these sample sizes are sufficient. For qualitative data, please indicate whether data saturation was considered, and what criteria were used to decide that no further sampling was needed.

Data collection

Provide details about the data collection procedure, including the instruments or devices used to record the data (e.g. pen and paper. computer, eye tracker, video or audio equipment) whether anyone was present besides the participant(s) and the researcher, and whether the researcher was blind to experimental condition and/or the study hypothesis during data collection.

Timing

Indicate the start and stop dates of data collection. If there is a gap between collection periods, state the dates for each sample cohort.

Data exclusions

If no data were excluded from the analyses, state so OR if data were excluded, provide the exact number of exclusions and the rationale behind them, indicating whether exclusion criteria were pre-established.

Non-participation State how many participants dropped out/declined participation and the reason(s) given OR provide response rate OR state that no participants dropped out/declined participation.

Randomization

\section{Ecological, evolutionary \& environmental sciences study design}

All studies must disclose on these points even when the disclosure is negative. 
Research sample

Sampling strategy

Data collection

Timing and spatial scale

Data exclusions

Reproducibility

Randomization

Blinding
Describe the research sample (e.g. a group of tagged Passer domesticus, all Stenocereus thurberi within Organ Pipe Cactus National Monument), and provide a rationale for the sample choice. When relevant, describe the organism taxa, source, sex, age range and any manipulations. State what population the sample is meant to represent when applicable. For studies involving existing datasets, describe the data and its source.

Note the sampling procedure. Describe the statistical methods that were used to predetermine sample size OR if no sample-size calculation was performed, describe how sample sizes were chosen and provide a rationale for why these sample sizes are sufficient.

Describe the data collection procedure, including who recorded the data and how.

Indicate the start and stop dates of data collection, noting the frequency and periodicity of sampling and providing a rationale for these choices. If there is a gap between collection periods, state the dates for each sample cohort. Specify the spatial scale from which the data are taken

If no data were excluded from the analyses, state so OR if data were excluded, describe the exclusions and the rationale behind them indicating whether exclusion criteria were pre-established.

Describe the measures taken to verify the reproducibility of experimental findings. For each experiment, note whether any attempts to repeat the experiment failed $O R$ state that all attempts to repeat the experiment were successful.

Describe how samples/organisms/participants were allocated into groups. If allocation was not random, describe how covariates were controlled. If this is not relevant to your study, explain why

Describe the extent of blinding used during data acquisition and analysis. If blinding was not possible, describe why OR explain why blinding was not relevant to your study.

Did the study involve field work? $\square$ Yes $\square$ No

Field work, collection and transport

Field conditions

Location

Access and import/export

Disturbance
Describe the study conditions for field work, providing relevant parameters (e.g. temperature, rainfall).

State the location of the sampling or experiment, providing relevant parameters (e.g. latitude and longitude, elevation, water depth).

Describe the efforts you have made to access habitats and to collect and import/export your samples in a responsible manner and in compliance with local, national and international laws, noting any permits that were obtained (give the name of the issuing authority, the date of issue, and any identifying information).

\section{Reporting for specific materials, systems and methods}

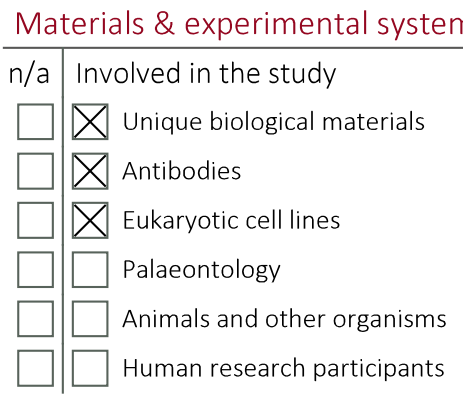

\begin{tabular}{l|l} 
Methods \\
\hline n/a Involved in the study \\
$\square$ ChIP-seq \\
$\square$ Flow cytometry \\
$\square$ MRI-based neuroimaging
\end{tabular}

\section{Unique biological materials}

Policy information about availability of materials

Obtaining unique materials The cleaved Ebola virus glycoprotein used in this study is available from the authors upon reasonable request.

\section{Antibodies}


macaque antibody and was recombinantly expressed as an IgG. A goat anti-mouse IgG-HRP from Santa Cruz Biotechnologies (sc-2005) was used as an ELISA secondary.

Validation

This is a structural biology paper. The antibody was complexed with the Ebola virus glycoprotein and this structure was solved by x-ray crystallography.

\section{Eukaryotic cell lines}

Policy information about cell lines

Cell line source(s)

Drosophila melanogaster S2, ExpiCHO

Authentication

none of the cell lines used were authenticated

Mycoplasma contamination

the cell lines were not tested for mycoplasma contamination

Commonly misidentified lines

(See $\underline{I C L A C}$ register)

Name any commonly misidentified cell lines used in the study and provide a rationale for their use.

\section{Palaeontology}

Specimen provenance
$\begin{aligned} & \text { Provide provenance information for specimens and describe permits that were obtained for the work (including the name of the } \\ & \text { issuing authority, the date of issue, and any identifying information). }\end{aligned}$
Specimen deposition
Dating methods Indicate where the specimens have been deposited to permit free access by other researchers.
$\begin{aligned} & \text { If new dates are provided, describe how they were obtained (e.g. collection, storage, sample pretreatment and measurement), } \\ & \text { where they were obtained (i.e. lab name), the calibration program and the protocol for quality assurance OR state that no new } \\ & \text { dates are provided. }\end{aligned}$

Tick this box to confirm that the raw and calibrated dates are available in the paper or in Supplementary Information.

\section{Animals and other organisms}

Policy information about studies involving animals; ARRIVE guidelines recommended for reporting animal research

Laboratory animals

Wild animals

Field-collected samples
For laboratory animals, report species, strain, sex and age on state that the study did not involve laboratory animals.

Provide details on animals observed in or captured in the field; report species, sex and age where possible. Describe how animals were caught and transported and what happened to captive animals after the study (if killed, explain why and describe method; if released, say where and when) OR state that the study did not involve wild animals.

For laboratory work with field-collected samples, describe all relevant parameters such as housing, maintenance, temperature photoperiod and end-of-experiment protocol OR state that the study did not involve samples collected from the field.

\section{Human research participants}

Policy information about studies involving human research participants

Population characteristics

Describe the covariate-relevant population characteristics of the human research participants (e.g. age, gender, genotypic information, past and current diagnosis and treatment categories). If you filled out the behavioural \& social sciences study design questions and have nothing to add here, write "See above."

Recruitment

Describe how participants were recruited. Outline any potential self-selection bias or other biases that may be present and how these are likely to impact results.

\section{ChIP-seq}

Data deposition

$\square$ Confirm that both raw and final processed data have been deposited in a public database such as $\underline{\text { GEO }}$.

$\square$ Confirm that you have deposited or provided access to graph files (e.g. BED files) for the called peaks.

\section{Data access links}

May remain private before publication.

Files in database submission

Genome browser session

(e.g. UCSC)
For "Initial submission" or "Revised version" documents, provide reviewer access links. For your "Final submission" document, provide a link to the deposited data.

Provide a list of all files available in the database submission. 
Provide a link to an anonymized genome browser session for "Initial submission" and "Revised version" documents only, to enable peer review. Write "no longer applicable" for "Final submission" documents.

Methodology

Replicates

Sequencing depth

Describe the experimental replicates, specifying number, type and replicate agreement.

Describe the sequencing depth for each experiment, providing the total number of reads, uniquely mapped reads, length of reads and whether they were paired-or single-end.

Antibodies

Describe the antibodies used for the ChIP-seq experiments; as applicable, provide supplier name, catalog number, clone name, and lot number.

Peak calling parameters

Specify the command line program and parameters used for read mapping and peak calling, including the ChIP, control and index files used.

Data quality

Describe the methods used to ensure data quality in full detail, including how many peaks are at FDR 5\% and above 5-fold enrichment.

Software

Describe the software used to collect and analyze the ChIP-seq data. For custom code that has been deposited into a community repository, provide accession details.

\section{Flow Cytometry}

Plots

Confirm that:

$\square$ The axis labels state the marker and fluorochrome used (e.g. CD4-FITC).

$\square$ The axis scales are clearly visible. Include numbers along axes only for bottom left plot of group (a 'group' is an analysis of identical markers).

$\square$ All plots are contour plots with outliers or pseudocolor plots.

$\square$ A numerical value for number of cells or percentage (with statistics) is provided.

Methodology

Sample preparation

Describe the sample preparation, detailing the biological source of the cells and any tissue processing steps used.

Instrument

Identify the instrument used for data collection, specifying make and model number.

Software

Describe the software used to collect and analyze the flow cytometry data. For custom code that has been deposited into a community repository, provide accession details.

Cell population abundance

Describe the abundance of the relevant cell populations within post-sort fractions, providing details on the purity of the samples and how it was determined.

Gating strategy

Describe the gating strategy used for all relevant experiments, specifying the preliminary FSC/SSC gates of the starting cell population, indicating where boundaries between "positive" and "negative" staining cell populations are defined.

Tick this box to confirm that a figure exemplifying the gating strategy is provided in the Supplementary Information.

\section{Magnetic resonance imaging}

Experimental design

Design type

Design specifications

Behavioral performance measures

Acquisition

Imaging type(s)

Field strength

Sequence \& imaging parameters
Indicate task or resting state; event-related or block design.

Specify the number of blocks, trials or experimental units per session and/or subject, and specify the length of each trial or block (if trials are blocked) and interval between trials.

State number and/or type of variables recorded (e.g. correct button press, response time) and what statistics were used to establish that the subjects were performing the task as expected (e.g. mean, range, and/or standard deviation across subjects).

Specify: functional, structural, diffusion, perfusion.

Specify in Tesla

Specify the pulse sequence type (gradient echo, spin echo, etc.), imaging type (EPI, spiral, etc.), field of view, matrix size, 
Sequence \& imaging parameters

Area of acquisition

Diffusion MRI

Used

Preprocessing

Preprocessing software

Normalization

Normalization template

Noise and artifact removal

Volume censoring

Statistical modeling \& inference

Model type and settings

Effect(s) tested

Specify type of analysis:

\section{Whole brain $\square$ ROI-based $\square$ Both}

Statistic type for inference (See Eklund et al. 2016)

\section{Correction}

Models \& analysis
$\mathrm{n} / \mathrm{a} \mid$ Involved in the study
$\square \square$ Functional and/or effective connectivity
$\square \square$ Graph analysis
$\square \mid \square$ Multivariate modeling or predictive analysis

Functional and/or effective connectivity

Report the measures of dependence used and the model details (e.g. Pearson correlation, partial correlation, mutual information).

Graph analysis

Multivariate modeling and predictive analysis
Provide detail on software version and revision number and on specific parameters (model/functions, brain extraction, segmentation, smoothing kernel size, etc.).

If data were normalized/standardized, describe the approach(es): specify linear or non-linear and define image types used for transformation OR indicate that data were not normalized and explain rationale for lack of normalization.

Describe the template used for normalization/transformation, specifying subject space or group standardized space (e.g. original Talairach, MNI305, ICBM152) OR indicate that the data were not normalized.

Describe your procedure(s) for artifact and structured noise removal, specifying motion parameters, tissue signals and physiological signals (heart rate, respiration).

Define your software and/or method and criteria for volume censoring, and state the extent of such censoring.

Specify type (mass univariate, multivariate, RSA, predictive, etc.) and describe essential details of the model at the first and second levels (e.g. fixed, random or mixed effects; drift or auto-correlation).

Define precise effect in terms of the task or stimulus conditions instead of psychological concepts and indicate whether ANOVA or factorial designs were used.

Specify voxel-wise or cluster-wise and report all relevant parameters for cluster-wise methods.

Describe the type of correction and how it is obtained for multiple comparisons (e.g. FWE, FDR, permutation or Monte Carlo).
Report the dependent variable and connectivity measure, specifying weighted graph or binarized graph, subject-or group-level, and the global and/or node summaries used (e.g. clustering coefficient, efficiency, etc.).

Specify independent variables, features extraction and dimension reduction, model, training and evaluation metrics. 